\title{
Systems Biology: An Information-Theoretic-Based Thermo-Statistical Approach
}

\author{
Marcus V. Mesquita, ${ }^{1}$ Áurea R. Vasconcellos, ${ }^{2}$, Roberto Luzzi ${ }^{2}$, and Sergio Mascarenhas ${ }^{3}$ \\ ${ }^{1}$ Instituto de Física, Universidade Federal do Ceará, 60455-760, Fortaleza, CE, Brazil \\ ${ }^{2}$ Instituto de Física 'Gleb Wataghin', Universidade Estadual de Campinas, Unicamp, 13083-970, Campinas, SP, Brazil \\ 3. Instituto de Estudos Avançados, Universidade de São Paulo, Campus de São Carlos, 13560-250 São Carlos, São Paulo, Brazil
}

Received on 22 May, 2003

\begin{abstract}
Systems Biology (system-level understanding in biological science), from the physical-chemical point of view, is involved with irreversible thermodynamics and nonlinear kinetic theory of open systems which are founded on nonequilibrium statistical mechanics. We describe a modern thermo-statistical approach for dealing with complex systems, in particular biological systems. We consider the case of a very peculiar complex behavior in open boson systems sufficiently away, from equilibrium, which appear to have large relevance in the functioning of biological systems. This is, on the one hand, the so-called Fröhlich-Bose-Einstein-like condensation leading in steady-state conditions to the emergence of a particular case of quantum-large-scale coherent ordering, of the type of a selforganizing-synergetic dissipative structure. Moreover, additional complexity emerges in the form of propagation, in this condensate, of signals (information) consisting of nearly undamped and undistorted, long-distance propagating, solitary waves (the pseudoparticle soliton). It can be accompanied by a so-called Fröhlich-Cherenkov cone of emission of polar vibrations, and it is also possible the formation of metastable states of the form of the so-called bioelectrets. These are phenomena apparently working in biological processes, which are presently gaining relevant status on the basis of eventually providing a large-scale quantum-coherent behavior in cytoskeletons of neurons and the conscious (non-computational) activity in the brain. Emphasis is centered on the quantum-mechanical-statistical irreversible thermodynamics of these open systems, and the informational characteristics of the phenomena. Ways for their experimental evidencing are pointed out and discussed.
\end{abstract}

\section{On the role of physics in biology}

What is Biophysics? For us, life is the most important phenomenon in Nature. It is also very complex, and in order to understand life and living processes several branches of science are needed. Biophysics uses biological and physical concepts for the study of life. One of the greatest physicist of the twentieth century, Erwin Schrödinger, wrote a beautiful little book which he named What is life? [1]. Though this book is now outdated in can be read with benefit by the modern scientist. Not only Physics but specially Biochemistry are essential to answer the question. So today, Biophysics is understood as a broad interdisciplinary area encompassing Biology, Physics, Biochemistry, mathematical and computational modelling, Theory of Information, and others. It is thus a very rich part of modern science with tremendous opportunities for basic and applied research. Physicists occasionally used models, intuitive theories and techniques to describe biology and life sciences. But also in the past, biologists, physicians, pharmacologists and other life scientists, rarely looked for physical concepts and instrumentation to help solve their problems. Until the mid-twentieth century Biology has been largely a descriptive field. It is only in the last half of the XX century that this gave place to a more complete, integrated approach, in which we can talk about Biophysics as an independent branch of Science [2].
A recent article in Science [3] had the seemingly taunting title of "Physicists advance into Biology", and a subtitle indicating that "physicists are [...] hoping that their mechanistic approach will yield new insight into biological systems": Both statements are open to questioning. The first one because there appears not to be an "invasion", but more precisely a "miscegenation" of sciences developing at the last decades of the second millennium and now going through the beginning of the XXI century. This has been foresighted and clearly stated by the renowned NobelPrize laureate Werner Heisenberg, who in 1970, in an article on the Wednesday October 6th. issue of the Süddeutsche Zeitung, wrote that "the characteristic feature of the coming development will surely consists in the unification of science, the conquest of the boundaries that have grown up historically between the different individual disciplines" [4]. In a sense this implies in a kind of "Renaissance" in the direction of an Aristotelian global philosophy of Natural Sciences. Even more interesting is the statement in the subtitle in [3], concerning the interdisciplinary aspects of Physics and Biology. What is most relevant to a theoretical physical approach to Biology is not the usual reductionistmechanicist-deterministic scheme of Physics, but an emerging scheme at a holistic-dynamicist-stochastic level. Citing the Nobel-Prize laureate Ilya Prigogine in a book in collaboration with Isabelle Stengers [5], "science centered around 
the basic conviction that at some level the world is simple and is governed by time-reversible fundamental laws. Today this appears as an excessive simplification. We may compare it to reduce buildings to piles of bricks [...] it is on the level of the building as a whole that we apprehend it as a creature of time, as a product of a culture, a society, a style." Moreover, Prigogine and Nicolis [6] wrote that "physics has emphasized stability and permanence. We now see that, at best, such qualification applies only to very limited aspects. Wherever we look, we discover evolutionary processes leading to diversification and increasing complexity." Prigogine and the so-called Brussel's school of thought, are among the pioneers of the nowadays referred-to as the highly interdisciplinary science of complexity. Complexity is regarded to be part of a frontier field in the particular science of Physics [7]. It is considered that the 1972 article in Science [8] by the Nobel-Prize laureate Philip W. Anderson constitutes one of the main "Manifests" on the subject (see also references [7-14]). Complex behavior in matter is nowadays a subject attracting an increasing interest. Complex Systems are not merely complicated (even though they could), but characterized by the fact of displaying highly coherent behavior involving the collective organization in a vast number of constituent elements. It is said that it is one of the universal miracles of Nature that huge assemblages of particles, subject only to the blind forces of nature, are nevertheless capable of organizing themselves into patterns of cooperative activity [7]. Complex behavior in matter can only arise in the nonlinear domain of the theory of dynamical systems [one of its founders being L. von Bertalanffy in the thirties [9]], since in the linear domain the principle of superposition of states cannot give rise to any unexpected behavior of a synergetic character. For thermodynamic systems, as the biological ones, coherent behavior is only possible in the nonlinear regime far from equilibrium, once in the linear (also referred as Onsagerian) regime around equilibrium synergetic organization is inhibited according to Prigogine's theorem of minimum entropy production $[6,10]$.

Systems Biology - as a branch of Dynamical Systems in Bertalanffy's style - deals with the problems of organization, with phenomena not resolvable into molecular or macromolecular events, and the dynamic interactions leading to complex functioning in living organisms. From the physico-chemical point of view it involves the irreversible thermodynamics of open systems and nonlinear kinetic theory, as already noticed. It has been stated that, system-level understanding has been a recurrent theme in biological science, gaining ample interest nowadays (see, eg. [15]) as becoming "fed" with basic information provided by the exploding molecular biology. Hence, there is now a golden opportunity for system-level analysis to be grounded in molecular-level understanding resulting in a continuous spectrum of knowledge, and while an understanding of genes and proteins continues to be important, the focus is on understanding the structure and dynamics of biological systems. [16]

Biological systems are complex systems by antonomasia, displaying an enormous variety of physico-chemical processes. Therefore, as it is the case, they present an also enormous number of rich and noticeable phenomena on the morphological, biochemical, biophysical, biomechanical, etc., levels. It ought to be emphasized that living organism are open systems driven (generally far) away from equilibrium and, then, the relevant area of Biophysics for their study is that of the nonlinear irreversible thermodynamics of open systems and its microscopic foundations (at the classical or quantal, nonlinear, memory-dependent, nonlocal, etc., levels) provided by nonequilibrium nonlinear statistical mechanics. These disciplines, irreversible thermodynamics of open systems and statistical mechanics of arbitrarily far-from-equilibrium systems, even though initiated in the XIX century by the great names of Boltzmann, Maxwell, and Gibbs, have been marred by conceptual and practical difficulties, but have recently shown vigorous development.

It is certainly a truism to say that the complicate heterogeneous spatial structure and functioning (temporal evolution) of living organisms, starting with the individual cell, set down quite difficult problems at the biophysical and biochemical levels of Biology. In recent decades a good amount of effort has in particular been devoted to some physicochemical aspects of biosystems, like, how to increase our knowledge of the chemical composition of life forms; to determine the structure of macromolecules, proteins, etc. (as noted in [3], understanding of structure is the first vital step, without which any further analysis run aground); to determine the reactions that lead to processes of sintetization of multiple components; to understand the mechanisms and codes required to determine the structure of proteins; and so on. Moreover, as already noticed, to consider living systems at the biophysical level we must be well aware of the fact that we are dealing with macroscopic open systems in nonequilibrium conditions. In other words, we observe macroscopic organization - at the spatial, temporal and functional levels - of the microscopic components of the system, namely, molecules, atoms, radicals, ions, electrons. The macroscopic behavior is of course correlated to the details of the microscopic structure. However, it must be further emphasized that this does not mean that knowing the microscopic details and their mechanistic laws, the redutionist scheme shall reveal the interesting macroscopic properties. Not only — as it is well known - is the number of microscopic states so huge that cannot be handled out, but still more important, and fundamental, is the relevant fact that macroscopic properties are expressed in terms of concepts that do not belong in mechanics, which are collective macroscopic effects. Hence, as already pointed out above, reductionist and deterministic methods of mechanics must be superseded - or, better to say, extended - to build a macrophysics, holistic in the sense of collective, and with both deterministic and chance characteristics. Some attempts in such direction have been developed with the introduction of approaches like Prigogine's dissipative structures [11], Fröhlich macroconcepts [12], Haken synergetics [13] and computer-modelling [17].

Which may be the theoretical approach in Physics to carry on a program to deal with at the microscopic as well as, at the same time, the all important, macroscopic levels of biosystems and their synergetic aspects? During the last 
decades this question concerning the theoretical description of the macroscopic behavior of dissipative open many-body systems in arbitrarily far-from-equilibrium conditions has been encompassed in a seemingly powerful, concise, and elegant formalism, established on sound basic principles. This is a nonequilibrium statistical ensemble formalism, accompanied with a nonlinear quantum kinetic theory, a response function theory for systems arbitrarily away from equilibrium, a statistical thermodynamics for dissipative systems, and a higher-order generalized hydrodynamics. This is the formalism used for the study of complex behavior in biological systems, mainly the so-called Fröhlich's effect and some other accompanying phenomena, as the long-distance propagation of nearly undamped and undistorted signals.

The formalism is based on a particular kind of scientific inference and the Bayesian approach to probability theory. According to Philip Anderson [18] the latter appears to be the most appropriate to use in science since it provides the degree of confidence that is consistent with keeping the idea that a proposition is correct when based on the fact to accept that other conditioning propositions are true: "These statistics are the correct way to do inductive reasoning from necessarily imperfect experimental data." This approach may be considered as an emerging paradigm in science, in the sense of the words of E. T. Jaynes that Science is information organized in a particular way. In the case of the natural sciences we can cite Jaynes [19]: "How shall we best think about Nature and most efficiently predict her behavior, given only our incomplete knowledge? [...] we need to see it, not as an example of the N-body equations of motion, but as an example of the logic of scientific inference, which by-passes all the details by going directly from our macroscopic information to the best macroscopic predictions that can be made from that information [...] Predictive Statistical Mechanics is not a physical theory, but a method of reasoning that accomplishes this by finding, not the particular things that the equations of motion say in any particular case, but the general things that they say, in 'almost-all' cases consistent with our information, for those are the reproducible things." Evidently, there remain quite difficult points to be cleared up, mainly how to determine in which extension this information depends on us, or up to what degree it is a "property" of Nature, or, better to say, of dynamical systems in general. Again, according to Jaynes, the question as to how the theoretically valid and pragmatically useful ways to apply Probability Theory in science was faced by Sir Harold Jeffreys [20,21], in the sense that he stated the general philosophy of what scientific inference is, and proceeded to develop a mathematical theory and its implementations. At the beginning of his book on Probability theory, Jeffreys maintains that the fundamental problem of scientific progress and a fundamental one of everyday life, is that of learning from experience. Knowledge obtained in this way is partly merely description of what we have already observed, but partly consists of making inferences from past experience to predict future experiences.

As already noticed, the nonequilibrium statistical ensemble formalism allows for the construction of a nonlinear quantum transport theory of a large scope and a re- sponse function theory for far-from-equilibrium systems. It also provides a thermodynamics of irreversible processes, dubbed as Informational Statistical Thermodynamics (IST, sometimes referred-to as Information-theoretic Thermodynamics). This IST was apparently pioneered by Hobson [22] after the publication of Jaynes' s seminal articles on the information-theoretic foundations of Statistical Mechanics [23, 24]. This irreversible statistical thermodynamics provides the foundations for the treatment of dissipative open macrosystems away (either near of far) from equilibrium [25]. This is the situation of biosystems, a result of the evident general feature that to function they require to have energy available which is provided by metabolic processes, that is, the open biosystem "feeds" on this energy and is driven away from equilibrium. A quite fundamental point is that the evolution of the system has associated a nonlinear kinetics. From the point of view of the macroscopic physics here involved (irreversible thermodynamics and kinetic theory), it consists in providing the description of nontrivial nonlinear effects that change in time and space and are maintained by means of a continuous energy supply.

We have already stressed the fact that the nonlinearity of the equations that describe the evolution in time of the macroscopic properties of the system is of fundamental relevance, and the source for complex behavior in matter. Complexity manifests itself in different situations involving this nonlinear domain of dynamical systems theory, and two relevant aspects are the nowadays fashionable deterministic chaos and self-organization in dissipative systems. This latter type of complex behavior in macroscopic system is the one that could apparently have enormous relevance in biosystems, in what is related to the origin of life, its functioning, and evolution. Two questions naturally arise concerning complex behavior in matter: (1) Which is the microscopic origin of complexity?, and (2) How can we deal theoretically with complex systems and be able to perform their rigorous analysis?

Here we describe an application of these ideas to a particular case of complex behavior in biosystems, with eventual large relevance in biopolymers (e.g. $\alpha$-chains in proteins), membranes, and microtubules in neuronal cells and its eventual connection with consciousnness as proposed by Roger Penrose [26]. Hence, and in conclusion of this Section, we may state that the results to be described, resulting from a promising particularly successful marriage of nonlinear nonequilibrium Statistical Thermodynamics and Biology, lead us to paraphrase Herbert Fröhlich saying that it is particularly auspicious to see that biological systems may display complex behavior describable in terms of appropriate physical concepts.

\section{General Considerations on Com- plexity in Boson Systems}

More than thirty years have elapsed since the renowned late Herbert Fröhlich first presented his concept of long-range coherence in biological systems [27], a question presently in a process of strong revival providing an attractive and rel- 
evant field of research in Physics and Biology. According to Fröhlich, biophysical systems possessing longitudinal electric vibrational modes may display, under appropriate conditions, a collective phenomenon akin to a Bose-Einstein condensation - not in equilibrium but as a complex behavior consisting in the emergence of a dissipative structure in Prigogine's sense [11]. Fröhlich's results are based on the idea that active biological systems are open and very far from equilibrium and have considerable amounts of energy available, through metabolic processes, that cause nonlinear changes in molecules and larger biological subsystems. In 'Life as a Collective Phenomena' [28], F. Fröhlich (Herbert's son) expressed that if one thinks without preconceptions of collective phenomena in which the discrete constitutive individuals are modified in their behavior, and indeed in their constituting a large collective group where the whole is more than and different from a simple addition of its parts, living organisms would seem to be the ideal example. Such a hypothesis of biological explanation in terms of long-range coherence was originally suggested by Fröhlich at the first meeting of L'Institut de la Vie in 1967 [27].

In Fröhlich model vibrational-polar modes are excited by a continuous supply of energy pumped by an external source, while these modes interact with the surrounding medium acting as a thermal bath. The interplay of these two effects - pumping of energy substracting entropy from the system and dissipative internal effects adding entropy to the system - , may lead to the emergence of complex behavior in the system consisting in what can be called Fröhlich effect: Provided the energy supply is sufficiently large compared with the energy loss, the system attains a stationary state in which the energy that feeds the polar modes is channelled into the modes with the lowest frequencies. The latter largely increase their populations at the expenses of the other higher-in-frequency modes, in a way reminescent of a Bose-Einstein condensation [29]. This highly excited subset of modes may exhibit long-range phase correlations of an electret type [30].

Fröhlich's synchronous large-scale collective oscillations imply in intercellular microwave emissions which would constitute a non-chemical and non-thermal interaction between cells. These oscillations could therefore be revealed by detection of emissions of $\mathrm{GHz}$ or $\mathrm{THz}$ radiation. Such electromagnetic signals are of extremely low magnitude and the receiver technology to measure them was not available during Fröhlich's time. It is only now that the predicted signals could be detected by adapting technology that has been developed for space and astrophysical research. Hence, a whole new area of biophysics is now ready for investigation [31].

Earlier experiments looking after Fröhlich effect were not conclusive, but now - as notice above - a 'second generation' of experiments are becoming available. They require further improvement, but already some preliminary results are encouraging [31]: Some evidence of a non-thermal influence of coherent microwave radiation on the genome conformational state in $E$. coli has been reported, which may indicate that chromosomal DNA could be the target of $\mathrm{mm}$ microwave irradiation within this system. Also low inten- sity microwave irradiation of leukocytes results in a significant increase in biophoton emission in the optical range, the origin of which is thought to involve DNA. Also it is worth noticing the possible influence of the concept of biocoherence on the very particular dipolar system which is water. It can be considered the possibility that biological water might itself support coherent dipolar excitations extending over mesoscopic regions; thus water, instead of being a passive space-filling solvent would be rised to an important singular position whose full significance has yet to be elucidated.

Nonbiological implications of Fröhlich effect could also be far-reaching. It can be mentioned some connection with homeopathy and atmospheric aerosol physics [31]. Regarding the latter, sunlight-pumped Fröhlich-like coherent excitations may play a role in producing anomalies in the spectrum of light absorption [32]. At this point we may mention a public safety concern, namely, the influence and eventual deleterious effects of mobile phones in close proximity to the head of the user as a result of the action of microwaves on the biological material.

Moreover, we call the attention to an important additional aspect of Fröhlich effect in connection with the longrange propagation of signals in biological and nonbiological materials. Such signals are wavepackets consisting of Schrödinger-Davydov solitons [33] which are a dynamical consequence of the same nonlinearities which are responsible for Fröhlich effect. It can be shown that the solitary wave which, in biological as well as nonbiological systems, is strongly damped as a result of the usual dissipative effects, may propagate with weak decay travelling long distances when moving in the background provided by a steady-state Fröhlich's condensate [34]. There already exist cases where theory is seemingly validated by experiment. One in the medical area of diagnosis, ultrasound imaging, is related not to Fröhlich effect in polar vibrational systems, but in acoustical vibrational ones. Fröhlich effect can also follow in the latter case with the pumping source being an anntena emiting ultrasound signals. A Davydov soliton, differently of the regular dispersive sound wave, travels long distances nearly unaltered, what can be of particular interest for improving detection in ultrasonography [35]. An interesting additional complex behavior follows, consisting in that when the soliton propagates with a velocity larger than that of the group velocity of the normal vibrational modes there follows a phenomenon akin to Cherenkov effect in radiation theory, namely a large emission of phonons in two symmetric cones centered on the soliton; this allows to interpret the so-called X-waves in ultrasonography as this FröhlichCherenkov effect [35]. In what regards nonbiological materials, we first notice the case of the molecular polymer acetanilide - which is a good mimic of certain biopolymers - where Davydov soliton is evidenced in the infrared absorption spectrum. In this case is open to the experimenter to look for an indirect verification of formation of Fröhlich condensate, determining the lifetime of the soliton (obtained via the Raman spectrum linewidth) when submitting the polar vibrational oscillations (the CO-stretching or Amide-I modes) to the action of an external pumping source (e.g. in- 
frared radiation) covering the frequencies of the dispersion relation of the vibrational modes [34].

Other example where Fröhlich's condensation and Davydov's soliton appear to be present is the case of the socalled 'Excitoner', meaning stimulated coherent emission of excitons created by random excitations, in a situation similar to the case of photons in a laser [36, 37]. In this case excitons, created in a semiconductor by an intense pulse of laser radiation, travel through the sample as a packet and are detected on the back of the sample. A weak signal in normal conditions of thermal excitation is largely enhanced when the system is pumped by a continuous external source of infrared radiation. The theory suggests the formation of a nonthermally excited Fröhlich condensate of excitons where a weakly damped Schrödinger-Davydov soliton is created, whose shape is in very good agreement with the experimental observation [38]. In summary, boson systems away from equilibrium may show a particularly noticeable complex behavior - Fröhlich effect; Davydov soliton; eventually Fröhlich-Cherenkov effect - , and the 'Excitoner', citing D. Snoke [36], is a phenomenon which may provide a new kind of light source, but only time and imagination can tell what new applications may arise from this novel effect.

Besides all these earlier aspects of Fröhlich's effect, quite recently there has been attempts to link it to a, say, quantum theory and the brain (see chapter 7 in Ref. [26]). Roger Penrose argues that computational procedures alone cannot adequately explain all the operational manifestations of human conscious understanding. It would be necessary to look for something different as the appropriate type of controlling mechanism - at least in the case of synaptic changes that might have some relevance to actual conscious activity. Such non-computational action must be the result of some reasonable large-scale quantum-coherent phenomenon, coupled in some subtle way to macroscopic behavior. As a first step, we must look for a genuine role for quantum coherence in cytoskeletal activity. Specifically, it would be required that there would be large-scale quantum-coherent behavior — of the like of the proposal put forward by Fröhlich - ocurring within the microtubules in the cytoskeleton of neurons. The suggestion is that this quantum activity should be non-computationally linked to a computational-like action (as for example argued by Hameroff et al. [39, 40]) taking place along microtubules (see Prologue in Ref. [26]).

Hence, Fröhlich's original ideas that such large-scale quantum states would be likely to occur in cell membranes, can be now extended — and perhaps more plausibly — introducing the possibility of presenting themselves in the microtubules, where then we can seek for coherent quantum behavior of this kind.

On the basis of this it appears to be appropriate to have an in-depth analysis of Fröhlich-Bose-Einstein-like condensation. This is so not only for the microscopic quantum mechanical aspects involved but, mainly, for the macroscopic aspects, that is to say, the particular nonequilibrium thermodynamics associated to the complex behavior of the open boson system providing the substrate for the phenomenon to occur. We must recall the quite important fact that biological systems are material systems which are open and working, in general, in far-from-equilibrium thermodynamic conditions.

Moreover, some additional related aspects of this complex system are present, which can give rise to associated phenomena of large relevance in biological systems, and mainly in the brain. They are the following:

1. Presence of ordered water (or biological water), which has extreme dielectric properties.

2. Formation of an ordered state of charge-density waves of the kind of an electret state.

3. The question of propagation of signals (information) at long distances, which possible in Fröhlich condensate, consisting of nearly undamped and undistorted Schrödinger-Davydov solitons.

4. Preferentially-directed emission of vibrational motion in a Fröhlich-Cherenkov cone.

Let us make some general previous comments on this, before proceeding with a review of results concerning the statistical thermodynamics of this engaging set of phenomena.

\subsection{Biological water.}

According to Penrose[26], "There is in the case of microtubules another matter of interest here, and this concerns the very nature of water. The tubes themselves appear to be empty - a curious and possibly significant fact in itself if we are looking to these tubes to provide for us the controlled conditions favorable to some kind of collective quantum oscillations. "Empty," here, means that they essentialy contain just water (without even dissolved ions). We might think that "water," with its randomly moving molecules, is not a sufficiently organized kind of structure for quantumcoherent oscillations to be likely to occur. However, the water that is found in cells is not at all like the ordinary water that is found in the oceans - disordered, with molecules moving about in an incoherent random way. Some of it and it is a controversial matter how much - exists in an ordered state. Such an ordered state of water may extend some $3 \mathrm{~nm}$ or more outwards from cytoskeletal surfaces. It seems not unreasonable to suppose that the water inside the microtubules is also of an ordered nature, and this would strongly favor the possibility of quantum-coherent oscillations within, or in relation to, these tubes."

\subsection{The electret state}

It has been conjectured the association of Fröhlich effect with the emergence of an induced ferroelectric metastable state of the electret type $[30,41]$. We recall that an electret is a piece of dielectric material exhibiting quasi-permanent electrical charges. The term "quasi-permanent" meaning that the time constants characteristics for the decay of the charge are much larger than the time periods over which studies are performed with the electret. 
Charge and polarization storage via the electret state has now been found in many biomaterials, where may have an important role in fundamental biophysical phenomena, as well as in biomedical applications. An important aspect of electret research in biophysics is that water bound to biopolymers in the so-called structured form (also called bound water or biowater) may also be induced into an electret state. The presence of electret states in biomaterials, mainly in biopolymers, seems to justify the usage of the term bioelectret.

It is worth noticing that the first electret was made with a material of biological origin, namely, carnauba wax from the carnauba palm tree of Brazil. This was the material originally used by Eguchi [42] to verify experimentally the theoretical proposal of Heaviside [43], who coined the name electret. Extensive pioneering investigations were due to Gross [44], and electret research gradually moved to involve many materials. Later on moved to the field of materials of biological origin like proteins, and the picture emerged that the electret effect may in fact be a universal property of biopolymers in general such as polypeptides, polynucleotides, polysacharides, etc. We are here adding to these possibilities the cytoskeleton.

\subsection{Propagation of signals}

The same nonlinearity in the kinetic equations, which are responsible for the complex behavior consisting of Fröhlich effect, give rise to the existence of a particular kind of excitation in this systems, namely, the Schrödinger-Davydov soliton. This is a quite convenient source for the propagation of signals, with the particular characteristic of being wholly reconstructed without distortion after collision processes.

Soliton is the name coined to describe a pulse-like nonlinear wave (the solitary wave referred to above) which emerges from a collision with a similar pulse having unchanged shape or speed. Its relevance in applied sciences has been described in a 1973 review paper by A. C. Scott $e t$ al. [45].

The original category of solitary waves consists in the one observed by the Scottish engineer Scott-Russell in August 1834 in an English water channel, and reported in a 1844 meeting of the British Society for the Advancement of Science [46]. During the second half of this century many other types of solitary waves have been associated with a number of physical situations in condensed matter physics. Several, seemingly, have a fundamental role in important technological areas of large relevance for contemporary society. Among them we may highlight the case of doped organic polymers with very large conductivity for, e. g., use in very light - almost two-dimensional (sheets) — batteries $[47,48,49]$, and the case of propagation of light in optical fibers [50]. Another example is that of the so-called Davydov's solitons [33, 51], which may have a quite relevant role in bioenergetics.

Davydov's theory has received plenty of attention, and a long list of results published up to the first half of 1992 are discussed in a comprehensive review due to A. C. Scott [52]. As pointed out in that review, one question concern- ing Davydov's soliton is that of its stability at normal physiological conditions, that is, the ability of the excitation to transport energy (and so information) at long distances in the living organism, in spite of the relaxation mechanisms that are expected to damp it out at very short (micrometers) distances. As we have shown, in fact Schrödinger-Davydov solitons are present in systems which follow Fröhlich modelling and, quite important, when travelling in Fröhlich condensate they provide undamped and undeformed ways to propagate signals at long distances $[34,53]$.

\subsection{Fröhlich-Cherenkov emission}

Further complex behavior is expected in this particular and notable system, that is, the possible emergence of a particular phenomenon, which we call Fröhlich-Cherenkov effect, consisting in that when the soliton is propagating with a velocity larger than the group velocity of the normal modes of vibration in the medium, a large number of phonons are emitted at a certain angle with the direction of propagation of the soliton [54].

We proceed next to present a revision of the main results on the subject, from a point of view of statistical thermodynamics.

\section{Fröhlich Effect in Polar Modes}

Let us consider a physical system modelling the conditions that lead to the emergence of Fröhlich effect. It is described in Fig. 1, where it is shown a particular biological system and the mechanical analog whose quantum mechanical statistical thermodynamics has been analyzed. What we do have is a periodic chain in which the polar vibrations of interest are the CO-stretching (Amide I) modes. The system is in interaction with the surroundings, a thermal bath modelled by an elastic-continuum-like medium. The reservoirs provide a homeostatic-like mechanism responsible for keeping the elastic continuum in equilibrium at temperature $T_{0}$ (say $300 \mathrm{~K}$ ). A source continuously pumps energy on the polar modes driving them out of equilibrium.

The Hamiltonian consists of the energy of the free subsystems, namely, that of the free vibrations, with $\omega_{\vec{q}}$ being their frequency dispersion relation ( $\vec{q}$ is a wave-vector running over the reciprocal-space Brillouin zone), and that of the thermal bath composed by oscillations with frequency dispersion relation $\Omega_{\vec{q}}$, with a Debye cut-off frequency $\Omega_{D}$. The interaction Hamiltonian $H_{I}$ contains the interaction of the system of polar vibrations with an external source (that pumps energy on the system), and the anharmonic interactions between both subsystems. The latter are composed of several contributions associated with quasi-particle (phonons) collisions involving the system and the thermal bath. 


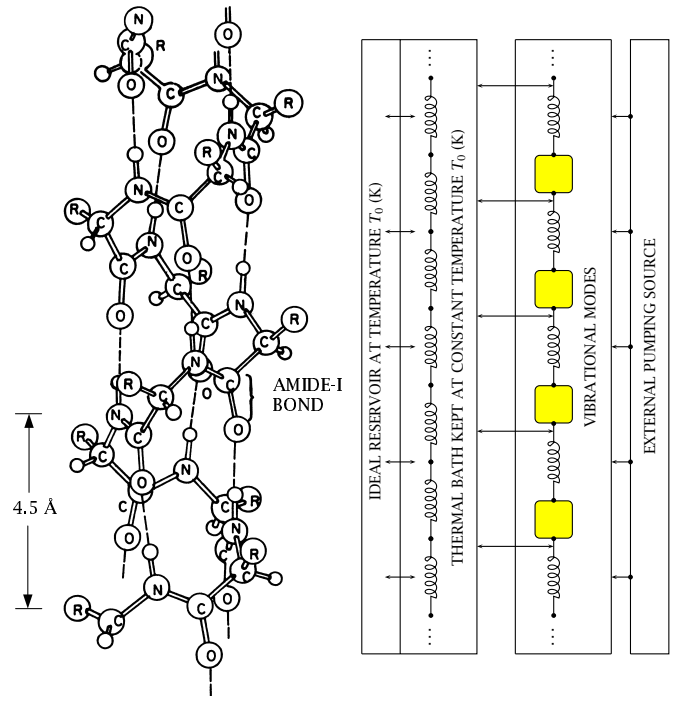

Figure 1. An atomic model of the $\alpha$-helix structure in a protein and a rough description of the mechanical model we used (After Ref. [29]).

For the quantum-mechanical statistical thermodynamic study of Fröhlich effect, whose results are reviewed here, we have resorted to an informational statistical thermodynamics based on the Nonequilibrium Statistical Operator Method (NESOM) [55-59] Besides providing microscopic foundations to phenomenological irreversible thermodynamics NESOM, also allows for the construction of a nonlinear generalized quantum transport theory - a farreaching generalization of Chapman-Enskog's and Mori's methods - which describes the evolution of the system at the macroscopic level in arbitrary nonequilibrium situations $[60,61,62]$. A most appropriated construction of the nonequilibrium ensemble formalism is based on a variational approach, consisting in the determination of the nonequilibrium statistical operator by means of Jaynes' principle of maximization of the quantity of uncertaintity of information (of Information Theory), usually referred-to as maximization of the informational entropy (MaxEnt for short). Thus from now on we will call the formalism as MaxEnt-NESOM, which is extensively described and discussed in Ref. [58] (see also Refs. [57] and [59]).

The next step consists in the choice, within the tenets of MaxEnt-NESOM, of the basic set of dynamical variables relevant for the present problem. Since we are dealing with excitation of vibrations in modes $\vec{q}$ (with energy $\hbar \omega_{\vec{q}}$ ), we need to introduce the number of excitations in each mode, $\hat{\nu}_{\vec{q}}=a_{\vec{q}}^{\dagger} a_{\vec{q}}$. The quantities $a_{\vec{q}}\left(a_{\vec{q}}^{\dagger}\right), b_{\vec{q}}\left(b_{\vec{q}}^{\dagger}\right)$ are as usual the annihilation (creation) operators of, respectively, normalmode vibrations in the system and bath in mode $\vec{q}$.

Moreover, once the formation of a coherent state is expected (Davydov's soliton), we must consider the field amplitudes $a_{\vec{q}}$ and $a_{\vec{q}}^{\dagger}$. Finally, since the thermal bath is taken as remaining constantly in a stationary state at a temperature $T_{0}$, via an efficient homeostatic mechanism, we introduce its Hamiltonian, $H_{B}$. The average of these quantities over the
NESOM nonequilibrium ensemble constituts the basic set of macrovariables, which we call

$$
\left\{\nu_{\vec{q}}(t),\left\langle a_{\vec{q}}^{\dagger} \mid t\right\rangle,\left\langle a_{\vec{q}} \mid t\right\rangle, E_{B}\right\}
$$

that is, they define Gibbs's space of nonequilibrium thermodynamic states. This is the thermodynamic state space in Informational Statistical Thermodynamics (IST for short) [25]. by

The basic thermodynamic macrovariables are then given

$$
\begin{aligned}
\nu_{\vec{q}}(t) & =\operatorname{Tr}\left\{\hat{\nu}_{\vec{q}} \varrho(t)\right\} ; \\
\left\langle a_{\vec{q}} \mid t\right\rangle & =\operatorname{Tr}\left\{a_{\vec{q}} \varrho(t)\right\} ; \\
\left\langle a_{\vec{q}}^{\dagger} \mid t\right\rangle & =\left\langle a_{\vec{q}} \mid t\right\rangle^{*}=\operatorname{Tr}\left\{a_{\vec{q}}^{\dagger} \varrho(t)\right\} ; \\
E_{B} & =\operatorname{Tr}\left\{\hat{H}_{B} \varrho(t)\right\},
\end{aligned}
$$

where $\varrho(t)$ is the nonequilibrium statistical operator in the present case, including the time-dependent part associated with the nonequilibrium macrostate of the system of interest (which is evolving in time) and the time-constant canonical distribution of the bath in equilibrium at temperature $T_{0}$. Hence $E_{B}$ is time independent since it depends only on the latter; $\mathrm{Tr}$ indicates as usual the trace operation.

We recall that the nonequilibrium thermodynamic state of the system, which is characterized by the set of macrovariables of Eqs. (2), can also - alternatively and completely - be characterized by the Lagrange multipliers that the variational MaxEnt-NESOM introduces. They constitute a set of intensive nonequilibrium thermodynamic variables, which we designate by

$$
\left\{F_{\vec{q}}(t), f_{\vec{q}}(t), f_{\vec{q}}^{*}(t), \beta_{0}\right\},
$$

where $\beta_{0}=\left(k_{B} T_{0}\right)^{-1}$, since the thermal bath remains in a stationary state at fixed temperature $T_{0}, k_{B}$ is Boltzmann universal constant. The nonequilibrium statistical operator depends on the dynamical variables $\hat{\nu}_{\vec{q}}, a_{\vec{q}}, a_{\vec{q}}^{\dagger}$, and $\hat{H}_{0 B}$ (the latter being the Hamiltonian of the stationary thermal bath) and on the Lagrange multipliers (nonequilibrium intensive thermodynamic variables) of Eq. (3).

Let us now consider the equations of evolution for the basic variables. Since $\beta_{0}$ is assumed to be constant in time, and so is $E_{B}$, we are simply left to calculate the equations of evolution for the population of the vibrational modes, $\nu_{\vec{q}}(t)$, and of the amplitude $\left\langle a_{\vec{q}} \mid t\right\rangle$ and its complex conjugate. As noticed, these equations are derived resorting to the nonlinear quantum generalized transport theory that the MaxEntNESOM provides. It was introduced as an approximate treatment, however appropriate for the present case since the anharmonic interactions are weak, consisting the so-called second-order approximation in relaxation theory, SOART for short [60]. It is usually referred to as the quasi-linear theory of relaxation [63], which is a Markovian approximation involving only the second order in the interaction strengths [60].

The calculation shows that, because of the symmetry properties of the system and the selected choice of basic 
variables, several contributions in MaxEnt-NESOM-SOART vanish in this case: The surviving ones correspond to the Golden Rule of quantum mechanics averaged over the nonequilibrium ensemble [29]. In compact form, the one for the population is

$$
\frac{d}{d t} \nu_{\vec{q}}(t)=I_{\vec{q}}+\sum_{j=1}^{5} J_{\vec{q}_{(j)}}(t)+\zeta_{\vec{q}}(t)
$$

where the first term on the right hand side is the one associated with the pumping source, where $I_{\vec{q}}$ is then the rate of population increase that it generates, the five collision operators $J_{\vec{q}_{(i)}}(t)$ are those arising out of the anharmonic interactions. The first two terms correspond to collisional events involving a single polar vibration and two of the bath, and give rise to a pure dissipative term which takes the form

$$
J_{\vec{q}_{(1)}}(t)+J_{\vec{q}_{(2)}}(t)=-\frac{1}{\tau_{\vec{q}}}\left[\nu_{\vec{q}}(t)-\nu_{\vec{q}}^{(0)}\right]
$$

where $\nu_{\vec{q}}^{(0)}$ is the population in equilibrium at temperature $T_{0}$, and $\tau_{\vec{q}}$ plays the role of a relaxation time. The other terms, $J_{\vec{q}_{(3)}}(t), J_{\vec{q}_{(4)}}(t)$, and $J_{\vec{q}_{(5)}}(t)$ are those which are nonlinear in the populations of the polar modes (and then can be the source of complexity). The first two are those responsible for the so-called Fröhlich effect, as a result that they account for, through the nonlinear terms, of the transfer of energy to the polar modes lowest in frequency. In fact, they contain nonlinear contributions proportional to

$$
\sum_{\vec{q}^{\prime}}\left|V_{\vec{q} \vec{q}^{\prime}}^{(2)}\right|^{2} \nu_{\vec{q}}(t) \nu_{\vec{q}^{\prime}}(t)\left[\delta\left(\Omega_{\vec{q}-\vec{q}^{\prime}}-\omega_{\vec{q}^{\prime}}+\omega_{\vec{q}}\right)-\delta\left(\Omega_{\vec{q}-\vec{q}^{\prime}}+\omega_{\vec{q}^{\prime}}-\omega_{\vec{q}}\right)\right],
$$

where $V_{\vec{q} \vec{q}^{\prime}}^{(2)}$ is the matrix element for the interaction (coupling strength), and we may notice that for modes $\vec{q}^{\prime}$ such that $\omega_{\vec{q}^{\prime}}>\omega_{\vec{q}}$ the energy conservation as required by the first delta function is satisfied, while this is not possible for the second. Hence, this nonlinear contribution tends to increase the population in mode $\vec{q}$ at the expenses of the other modes higher in frequency. Reciprocally, for $\omega_{\vec{q}^{\prime}}<\omega_{\vec{q}}$, the mode $\vec{q}$ transfers energy to the modes lower in frequency. Moreover, in Eq. (4) the term $\zeta_{\vec{q}}(t)$ acts as a source coupling the populations of the vibrational modes to the amplitudes of the expected coherent excitation (Davydov's soliton as shown $a$ posteriori).

On the other hand, the equations of evolution for the field amplitudes are

$$
\begin{aligned}
\frac{\partial}{\partial t}\left\langle a_{\vec{q}} \mid t\right\rangle & =-\mathrm{i} \tilde{\omega}_{\tilde{\mathrm{q}}}\left\langle\mathrm{a}_{\tilde{q}} \mid \mathrm{t}\right\rangle-\Gamma_{\tilde{\mathrm{q}}}\langle\mathrm{a} \tilde{\mathrm{q}} \mid \mathrm{t}\rangle+\Gamma_{\tilde{\mathrm{q}}}\left\langle\mathrm{a}_{\tilde{q}} \mid \mathrm{t}\right\rangle^{*}-\mathrm{iW}_{\tilde{\mathrm{q}}}\left\langle\mathrm{a}_{\tilde{\mathrm{q}}} \mid \mathrm{t}\right\rangle^{*}+ \\
& +\sum_{\vec{q}_{1} \vec{q}_{2}} R_{\vec{q}_{1} \vec{q}_{2}}\left\langle a_{\vec{q}_{1}} \mid t\right\rangle\left\langle a_{\vec{q}_{2}}^{\dagger} \mid t\right\rangle\left(\left\langle a_{\vec{q}-\vec{q}_{1}+\vec{q}_{2}} \mid t\right\rangle+\left\langle a_{-\vec{q}+\vec{q}_{1}-\vec{q}_{2}}^{\dagger} \mid t\right\rangle\right), \\
\frac{\partial}{\partial t}\left\langle a_{\vec{q}}^{\dagger} \mid t\right\rangle & =\text { the c.c. of the r.h.s. of Eq. (7a), }
\end{aligned}
$$

where $\tilde{\omega}_{\vec{q}}=\omega_{\vec{q}}+W_{\vec{q}}, W_{\vec{q}}$ is a term of renormalization of frequency which will not be of interest in the following analysis, and the lengthy expression for $R_{\vec{q}_{1} \vec{q}_{2}}$ is given elsewhere [53] (its detailed expression is unnecessary for the analysis here). Finally, $\Gamma_{\vec{q}}(t)$, which has a quite relevant role in what follows, is given by

$$
\begin{aligned}
\Gamma_{\vec{q}}(t) & =\frac{1}{2} \tau_{\vec{q}}^{-1}(t)+\frac{4 \pi}{\hbar^{2}} \sum_{\vec{q}^{\prime}}\left|V_{\vec{q} \vec{q}^{\prime}}^{(2)}\right|^{2}\left[1+\nu_{\vec{q}^{\prime}}+\nu_{\vec{q}-\vec{q}^{\prime}}^{B}\right] \delta\left(\Omega_{\vec{q}-\vec{q}^{\prime}}+\omega_{\vec{q}^{\prime}}-\omega_{\vec{q}}\right) \\
& -\frac{4 \pi}{\hbar^{2}} \sum_{\vec{q}^{\prime}}\left|V_{\vec{q} \vec{q}^{\prime}}^{(2)}\right|^{2}\left[\nu_{\vec{q}^{\prime}}-\nu_{\vec{q}-\vec{q}^{\prime}}^{B}\right] \delta\left(\Omega_{\vec{q}-\vec{q}^{\prime}}-\omega_{\vec{q}^{\prime}}+\omega_{\vec{q}}\right) \\
& +\frac{4 \pi}{\hbar^{2}} \sum_{\vec{q}^{\prime}}\left|V_{\vec{q} \vec{q}^{\prime}}^{(2)}\right|^{2}\left[\nu_{\vec{q}^{\prime}}-\nu_{\vec{q}+\vec{q}^{\prime}}^{B}\right] \delta\left(\Omega_{\vec{q}+\vec{q}^{\prime}}-\omega_{\vec{q}^{\prime}}-\omega_{\vec{q}}\right)
\end{aligned}
$$


where $\nu_{\vec{p}}^{B}$ is the population of the phonons of the thermal bath in mode $\vec{p}$.

The coupled equations (7) contain linear and tri-linear terms. Ignoring the latter, the resulting linearized equation has as solutions the normal damped wave motion, proceeding with a renormalized frequency $\tilde{\omega}_{\vec{q}}$ and lifetime $\Gamma_{\vec{q}}^{-1}$. The complete equations, i.e. including the nonlinear terms, are of the Davydov's soliton type, but with damping, or more precisely, are nonlinear damped Schrödinger-like equations $[45,64]$.

The presentation above has clearly evidenced the presence in the kinetic equations for the populations of the polar modes of the nonlinearities which are responsible for Fröhlich's effect. Let us better characterize this. We recall the results of Refs. [29] and [65]: conservation of energy and momentum in the collisional events leads to the relevant fact that the polar modes are coupled in bundles involving a certain small number of modes; such number depends on the physical parameters of the system. Figure 2 illustrates a particular case when twelve modes are coupled (those six and other six symmetrical in Brillouin zone). It is clear the emergence of Fröhlich's effect at a (scaled) intensity of the external source of roughly $10^{3}$; the mode at the lowest frequency with population, $\nu_{1}$, greatly increases (note that the scale is logarithmic) at the expenses of the other modes higher in frequency. Here $\lambda$ is an open parameter measuring the intensity of the nonlinear coupling in the kinetic equations (for illustration we took $\lambda=1$ ).

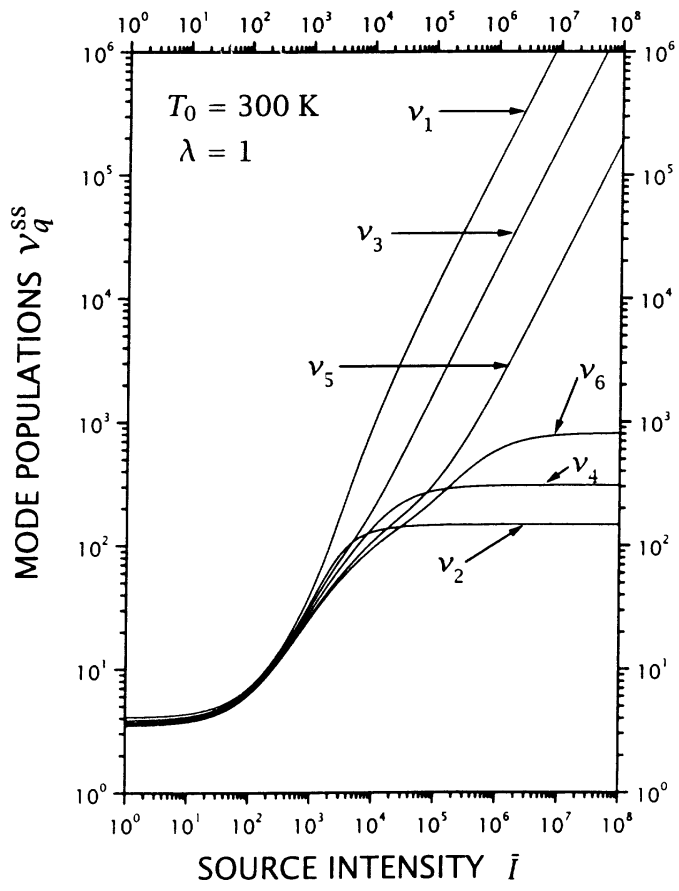

Figure 2. Populations of a set of modes in the steady state under a constant pumping intensity $\bar{I}$.

Evidently, there exist a large number of sets of twelve modes repeating this behavior, and then there is a collection of modes, those at the low frequency side of the frequency dispersion spectrum, which grow largely at the expenses of all the other modes in Brillouin zone. This is shown in Fig. 3, for a scaled intensity of the pumping source of $2.6 \times 10^{5}$, i.e. (see Fig. 2) above the threshold for the onset of Fröhlich's effect. For comparison we have drawn the case (diamond-line) when the nonlinear coupling is switched off and clearly is observed a normal behavior of similar increase in all the populations.

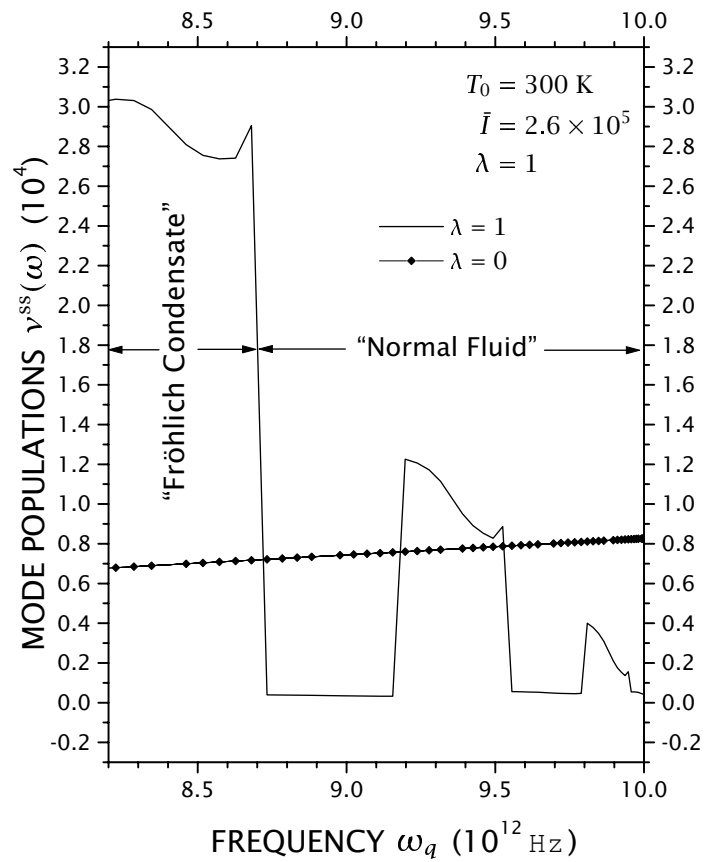

Figure 3. Populations of the modes in the steady state for $\bar{I}=$ $2.6 \times 10^{5}$, compared with the case of absence of nonlinear interactions.

In terms of the Lagrange multipliers of Eq. (4), the population in each mode $\vec{q}$ is given by

$$
\nu_{\vec{q}}(t)=\left[\exp \left\{F_{\vec{q}}(t)\right\}-1\right]^{-1}+\left|f_{\vec{q}}(t) / F_{\vec{q}}(t)\right|^{2},
$$

composed of a main contribution (resembling a Plancklike distribution) plus another one related to the amplitudes $\left(\left|\left\langle a_{\vec{q}} \mid t\right\rangle\right|^{2}\right)$ and small.

Now, the Lagrange parameter $F_{\vec{q}}(t)$ (intensive nonequilibrium thermodynamic variable) can alternatively be written in either of two forms, namely

$$
F_{\vec{q}}(t)=\beta_{0}\left[\hbar \omega_{\vec{q}}-\mu_{\vec{q}}(t)\right]
$$

introducing a so-called quasi-chemical potential per mode $\mu_{\vec{q}}(t)$, as done by Landsberg [66] and Fröhlich [27], and where $\beta_{0}=1 / k_{B} T_{0}$, or

$$
F_{\vec{q}}(t)=\beta_{\vec{q}}(t) \hbar \omega_{\vec{q}}=\frac{\hbar \omega_{\vec{q}}}{k_{B} T_{\vec{q}}^{*}(t)}
$$

introducing a quasitemperature per mode as it is done in the physics of semiconductors $[67,68]$ and in radiation theory [69]. 


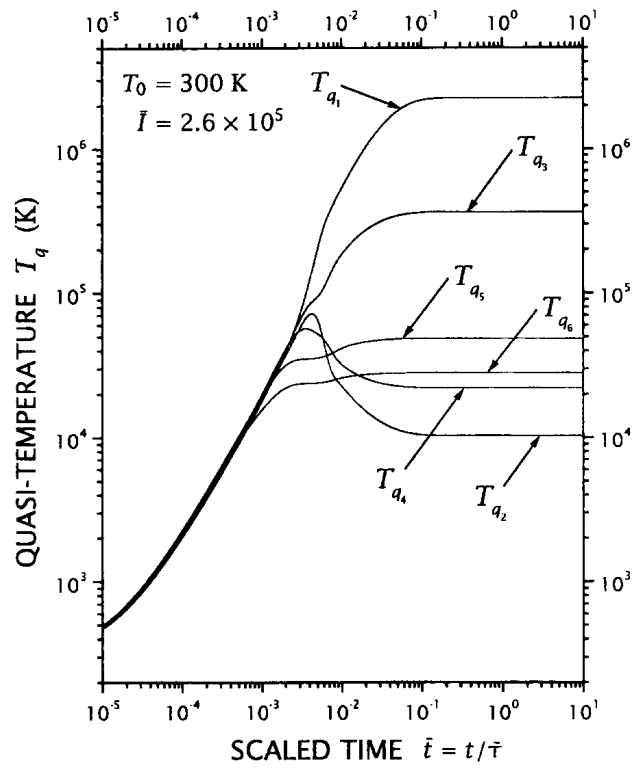

Figure 4. The quasitemperature associated to the set of modes in Fig. 2.

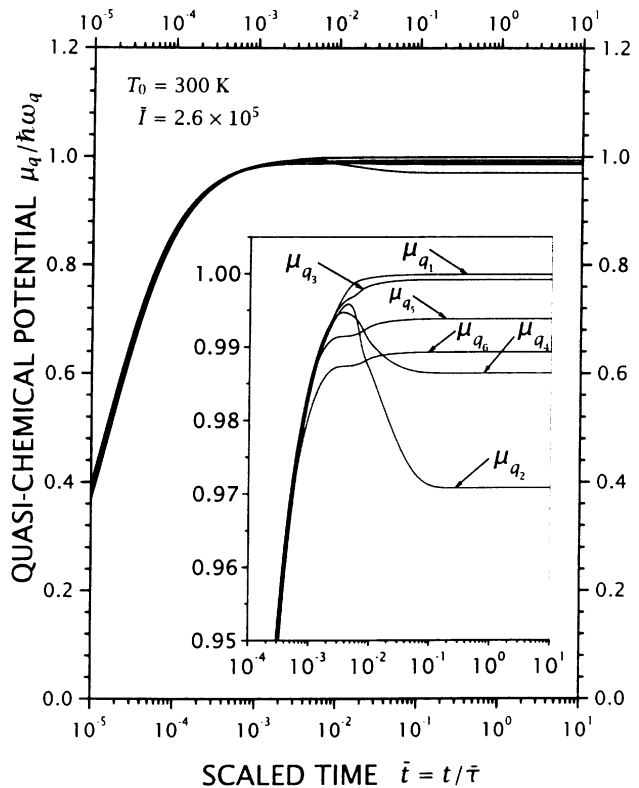

Figure 5. The quasi-chemical potential associated to the set of modes in Fig. 2.

In the case of the modes involved in Fig. 2, we are pre- senting in Fig. 4 the evolution in time of the quasitemperature of these modes, which keeps increasing until the steady state sets in. It is clear the large increase (note that the scale is logarithmic) of the quasitemperature of the mode lowest in frequency in the set. On the other hand, in Fig. 5 is presented the evolution in time of the quasi-chemical potentials, showing that $\mu_{1}$ tends to a steady-state value that comes quite near to the frequency $\omega_{1}$ (tends to it as the intensity of the source goes to infinity); hence, it ought to be noticed that the quasi-chemical potential does not coincide with the lowest frequency and therefore there is not a true analogy with a Bose-Einstein condensation. Thus, to refer Fröhlich's effect as a Bose-Einstein-like condensation is a kind of freedom of speech.

Having thus clearly characterized Fröhlich's effect in the context of a NESOM-based nonlinear quantum kinetic theory, we proceed to describe the relevant associated phenomenon of solitary waves propagation.

\section{Undamped and Undeformed Sig- nals}

We consider now the propagation of coherent signals in Fröhlich's condensate, that is proceeding to the analysis of the equations of evolution for the polar modes amplitudes, as given by Eqs. (7a) and (7b).

We introduce a representation in direct space, defining the averaged (over the nonequilibrium ensemble) field operator

$$
\psi(x, t)=\sum_{\vec{q}}\left\langle a_{\vec{q}} \mid t\right\rangle \mathrm{e}^{\mathrm{iqx}} .
$$

for linear propagation along the, say, $x$ direction in bulk or along the one-dimensional polymer. At this point we consider a first type of analysis, specifying the vibrational modes as being of the class of longitudinal polar-modes we have been considering. Their frequency dispersion relation can be approximated (to a good degree of accuracy) by the parabolic-law $\omega_{\vec{q}}=\omega_{0}-\alpha q^{2}$, where $\omega_{0}$ and $\alpha$ are constants, standing for the frequency at the zone center (the maximum one) and the curvature at this centre, respectively. Next, using Eqs. (7), after neglecting the coupling terms with the conjugated amplitude (what can be shown to be the case when we introduce from the outset a truncated Hamiltonian in the so-called Rotating Wave Approximation [70]), it follows that the average field amplitude satisfies the equation

$$
\begin{aligned}
\mathrm{i} \hbar \frac{\partial}{\partial \mathrm{t}} \psi(\mathrm{x}, \mathrm{t}) & =\left(\hbar \omega_{0}+\hbar \alpha \frac{\partial^{2}}{\partial x^{2}}\right) \psi(x, t)-\mathrm{i} \hbar \int_{0}^{\mathrm{L}} \frac{\mathrm{dx}^{\prime}}{\mathrm{L}} \Gamma\left(\mathrm{x}-\mathrm{x}^{\prime}\right) \psi\left(\mathrm{x}^{\prime}, \mathrm{t}\right)+ \\
& +\int_{0}^{L} \frac{d x^{\prime}}{L} \int_{0}^{L} \frac{d x^{\prime \prime}}{L} \mathcal{R}\left(x-x^{\prime}, x-x^{\prime \prime}\right) \psi\left(x^{\prime}, t\right) \psi\left(x^{\prime \prime}, t\right) \psi^{*}(x, t),
\end{aligned}
$$


where $\Gamma$ and $\mathcal{R}$ are the back-transforms to direct space of $\Gamma_{\vec{q}}$ in Eq. (8) and $R_{\vec{q}_{1} \vec{q}_{2}}$ in Eq. (7a), and $L$ is the length of the sample. Moreover, we have taken a time-independent population $\nu_{\vec{q}}$, that is, according to Eq. (4) it is either the equilibrium distribution at temperature $T_{0}$ when no external pumping source is present (i. e. $I_{\vec{q}}\left(\omega_{\vec{q}}\right)=0$ ), or when in the presence of a constant pumping source leading, after a short transient has elapsed, to a steady state.

Equation (13) is a nonlinear Schrödinger-type equation with damping [64]. Introducing a local approximation, that is, neglecting space correlations, after using the expressions

$$
\begin{array}{rlr}
\mathcal{R}\left(x-x^{\prime}, x-x^{\prime \prime}\right) & =\hbar G \delta\left(x-x^{\prime}\right) \delta\left(x-x^{\prime \prime}\right),(14 \mathrm{a}) \\
\Gamma\left(x-x^{\prime}\right) & =\gamma_{s} \delta\left(x-x^{\prime}\right),
\end{array}
$$

it follows that Eq. (13) becomes

$$
\begin{gathered}
\mathrm{i} \frac{\partial \psi(\mathrm{x}, \mathrm{t})}{\partial \mathrm{t}}-\left(\omega_{0}-\mathrm{i} \gamma_{\mathrm{s}}\right) \psi(\mathrm{x}, \mathrm{t}) \\
-\alpha \frac{\partial^{2}}{\partial x^{2}} \psi(x, t)-G|\psi(x, t)|^{2} \psi(x, t)=0,
\end{gathered}
$$

Equations (7) and (15) are of the form of the equations derived by Davydov in an alternative way, but the present thermodynamic treatment is clearly showing the presence of damping effects. In equilibrium conditions at temperature $300 \mathrm{~K}$, the damping constants have values corresponding to lifetimes in the order of a few picoseconds. For the case of a Gaussian signal impinged at the beginning of the polymer chain, which can be approximated to a good degree of accuracy by a hyperbolic secant shape, and next using the Inverse Scattering Method [71] it is obtained the solution

$$
\psi(x, t)=\mathcal{A} \exp \left\{\mathrm{i}\left[\frac{v}{2 \alpha} \mathrm{x}-\left(\omega_{\mathrm{s}}-\mathrm{i} \gamma_{\mathrm{s}}\right) \mathrm{t}-\frac{\theta}{2}\right]\right\} \operatorname{sech}\left[\mathcal{A}\left(\frac{|\mathrm{G}|}{2 \alpha}\right)^{1 / 2}(\mathrm{x}-v \mathrm{t})\right]
$$

where $\gamma_{s}$ is, evidently, the reciprocal lifetime of the excitation (taking $\gamma_{s}=0$ and $\omega_{0}=0$, Eq. (16) is the expression for Davydov's soliton in its original version [33, 51]), and we used $G=|G| \mathrm{e}^{\mathrm{i} \theta}$. Moreover,

$$
\omega_{s}=\omega_{0}-\frac{v^{2}}{4 \alpha}+\frac{|G| \mathcal{A}^{2}}{2},
$$

is the reciprocal period of the solitary wave and $\mathcal{A}$ and $v$ are an amplitude and a velocity of propagation fixed by the initial condition of excitation imposed by the external source.

Hence, it is proved the possible presence of Davydov's solitons in polymers, like the $\alpha$-helix protein in biological matter, but, we stress, of a damped character. The mechanism for the formation of the soliton is in this case interpreted as follows [52]: Vibrational energy of the COstretching (Amide-I) oscillators that is localized on the quasi-periodic helix acts - through a phonon coupling effect - to distort the structure of the helix. The helical distortion reacts - again through phonon coupling — to trap the Amide-I oscillation and prevents its dispersion in a selftrapping-like process.

Let us consider the experimental observation of this excitation. As already noticed, experimental observation is difficult in active biological materials. A way around this difficulty consists into the experimental study of polymers whose vibronic characteristics resemble those of biopolymers, and a quite favorable one is acetanilide. Experiments of infrared absorption in acetanilide showed an "anomalous" band in the IR spectrum, which was ascribed to a Davydov's soliton [72], and later on reproduced in other experiments, and also observed in Raman scattering experiments $[73,74,75,76,77]$.

We analyzed the experiment of Careri et. al [72], resorting to a response function theory consistently derived in the framework of MaxEnt-NESOM [57, 78, 79]. Without going into details (see $[34,80]$ ), the absorbance in the region of the CO-stretching mode has the expression

$$
\alpha(\omega)=\alpha_{n}(\omega)+\alpha_{s}(\omega)
$$

which describes two bands: one centered around $\omega_{\vec{q}}$, the frequency of the normal mode, $(\vec{q}$ being equal to the IR-photon wave-vector) and with intensity proportional to the population in equilibrium $\nu_{\vec{q}}$ at temperature $T_{0}$, since no external pump is present $\left[I_{\vec{q}}=0\right.$ in Eq. (4)] and the other is centered around $\omega_{s}$, the frequency of the soliton. The band widths are $\gamma_{n}=\tau_{0}^{-1}$ and $\gamma_{s}=\tau_{0}^{-1}+\mathcal{A}(|G| / 2 \alpha)^{1 / 2}$, where $\tau_{0}^{-1}$ is the one of Eq. (5), for $\vec{q}$ near the zone center. Let us consider the experiments of reference [72], and take, for example, the case of $T_{0}=80 \mathrm{~K}$; on the basis of the red shift of the band due to the soliton in relation to the normal CO-stretching band, that is, $\omega_{0}-\omega_{s} \approx 16 \mathrm{~cm}^{-1}$, and that $\gamma_{s}-\gamma_{n} \approx 3.6 \mathrm{~cm}^{-1}$, we find that $\mathcal{A}(|G| / 2 \alpha)^{1 / 2} \approx$ $2.3 \times 10^{6} \mathrm{~cm}^{-1}$ and $v \approx 3 \times 10^{4} \mathrm{~cm} \mathrm{~s}^{-1}$. The calculated spectra is shown as a full line in the lower part of Fig. 6, while the dots are experimental points, evidencing a satisfactory agreement; in this figure are also shown the spectra corresponding to $T_{0}=50 \mathrm{~K}$ and $20 \mathrm{~K}$, where again there is a good agreement between theory and experiment.

In Fig. 7 it is shown the propagation of the energy accompanying the soliton (proportional to $|\psi(x, t)|^{2}$ ) along a few picoseconds after the application of the initial Gaussianlike excitation. It is clearly evidenced the conservation of the shape characteristic of the soliton, but accompanied, as already described, with a decay in amplitude in the picosecond range. Hence, a pulse signal impinged on the system would be carried a few micrometers, since the velocity of propagation is $\approx 3 \times 10^{4} \mathrm{~cm} \mathrm{~s}^{-1}$. 


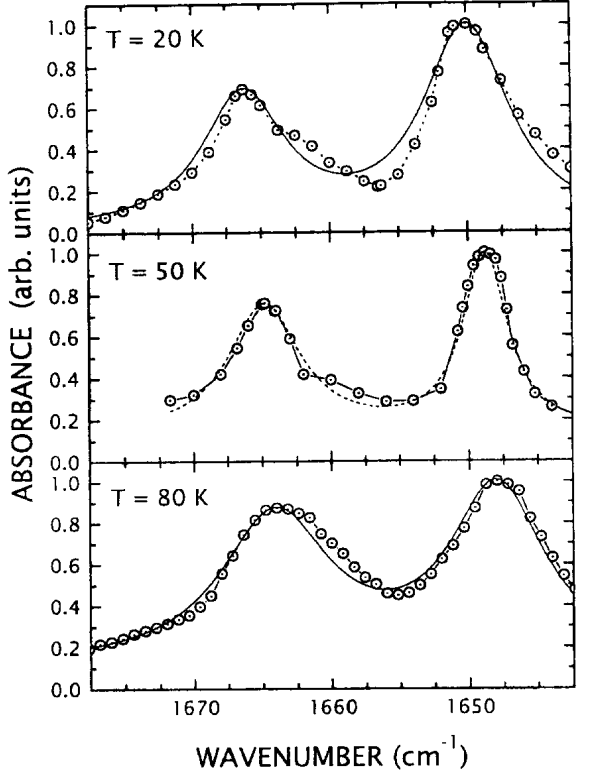

Figure 6. Infrared absorption spectra in acetanilide for three different values of temperature. Dotted curve is from the experimental data of reference [37], and the full curve the calculation in NESOMbased response function theory.

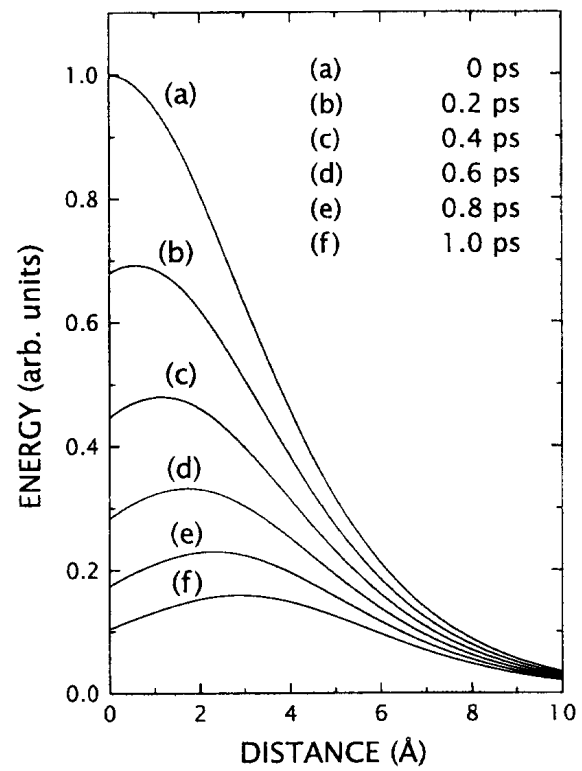

Figure 7. Optical vibrations: Profile of the soliton energy (proportional to $|\psi(x, t)|^{2}$ ) along a few picoseconds after application of the initial Gaussian-like perturbation (in the experimental conditions reported in Ref. [37] for $T=80 \mathrm{~K}$ ).

However, the situation may be substantially modified if the excitation propagates in a nonequilibrium background, namely the one provided by the presence of a constant pumping source $\left[I_{\vec{q}} \neq 0\right.$ in Eq. (4)] leading the system to a steady state, with populations, say, $\bar{\nu}_{\vec{q}}$, constant in time but much larger than the population in equilibrium, particularly in the Fröhlich's condensate described in the previous Section. As we have seen the solitary wave is a wave packet formed in terms of the amplitudes $\left\langle a_{\vec{q}} \mid t\right\rangle$, as indicated by Eq. (12), which follows the equation of evolution, Eq. (7), whose solution is Eq. (16). But Eq. (16) is a consequence of the Eq. (7a) for the amplitudes, where we can see that is present a damping with a characteristic lifetime $\Gamma_{\vec{q}}^{-1}$, given by Eq. (8). But it has a peculiar form, i.e. it is composed of a relaxation time $\tau_{\vec{q}}$ (associated to the decay of the polar vibrations producing thermal agitation in the surrounding media) plus a contribution associated to the presence of the nonlinear coupling terms in the equations of evolution. They depend on the populations of the polar modes and have the very peculiar characteristic that for the modes in the condensate, with increasing populations the quantity $\Gamma_{\vec{q}}$ decreases and then the lifetime of these modes increases. On the contrary, for the "normal" modes $\Gamma_{\vec{q}}$ increases and then the lifetime of these modes decreases.

It is quite interesting to note a close connection with Fröhlich's effect, in the sense that in the steady state, the population of the polar modes is given by

$$
\bar{\nu}_{\vec{q}} \simeq N_{\vec{q}} / 2 \Gamma_{\vec{q}}
$$

and precisely the fact that $\Gamma_{\vec{q}}$ decreases (and tends to zero with increasing intensity of the pumping source) for the modes in the condensate, these modes largely grow. Using the "two fluid" model suggested by Fig. 3, that is neglecting the minor differences between both types of modes with different $\vec{q}$, we can estimate the reciprocal of the lifetimes for the modes in the condensate (index one) and in the "normal" fluid (index nought) in terms of the intensity of the source, what is shown in Fig. 8 (notice the logarithmic scale).

Hence, we can write Eq. (12) as

$$
\psi(x, t)=\sum_{\vec{q} \in \mathcal{R}_{1}}\left\langle a_{\vec{q}} \mid t\right\rangle \mathrm{e}^{\mathrm{iqx}}+\sum_{\tilde{\mathrm{q}} \in \mathcal{R}_{0}}\left\langle\mathrm{a}_{\tilde{\mathrm{q}}} \mid \mathrm{t}\right\rangle \mathrm{e}^{\mathrm{iqx}},
$$

where $\mathcal{R}_{1}$ refers to the modes in the condensate and $\mathcal{R}_{0}$ in the "normal" fluid. But beyond the threshold for emergence of Fröhlich's effect the contribution from the modes in $\mathcal{R}_{0}$ decay rapidly (pico- to subpico-second scales), surviving with a very long lifetime the contribution of the modes in $\mathcal{R}_{1}$. Therefore, the soliton is composed of an undeformed wavepacket of these modes with a very long lifetime, i.e. that $\gamma_{S} \rightarrow 0$ in Eq. (16).

Therefore we do have the quite relevant result that once Fröhlich's condensate has been established, signals can propagate along systems showing such complex behavior (a macroscopic quantum-coherent state) as nearly undamped - and then traversing long distances - and unaltered that is they are neither deformed nor, more important, modified while crossing other signals. This is illustrated in Fig. 9. 


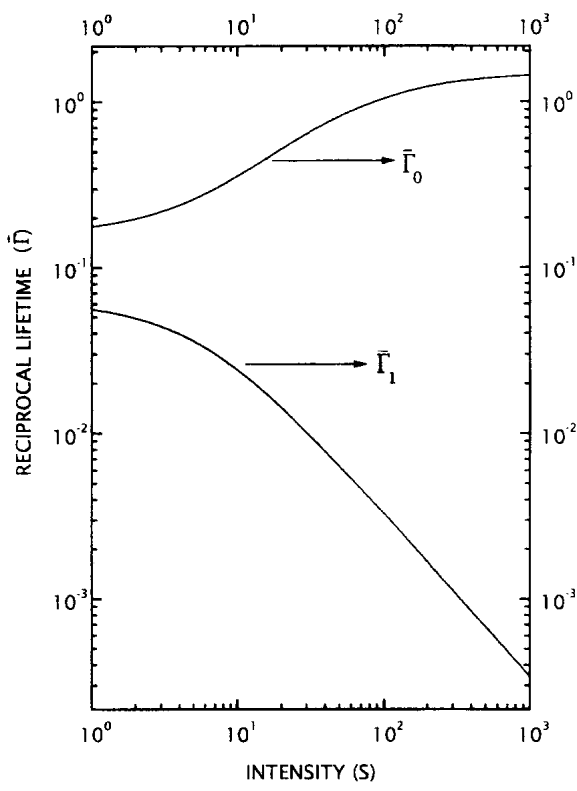

Figure 8. Reciprocal lifetime of the representative high frequency modes (index nought) and of the low frequency modes (index one) with increasing intensity of the source. Both $\bar{\Gamma}$ are in units of $\bar{\tau} \sim 0.4$ picoseconds, and the intensity $S$ in adimensional units, but such that $1 S$ corresponds to a pumping power of $1 \mu \mathrm{W}$ per mode.

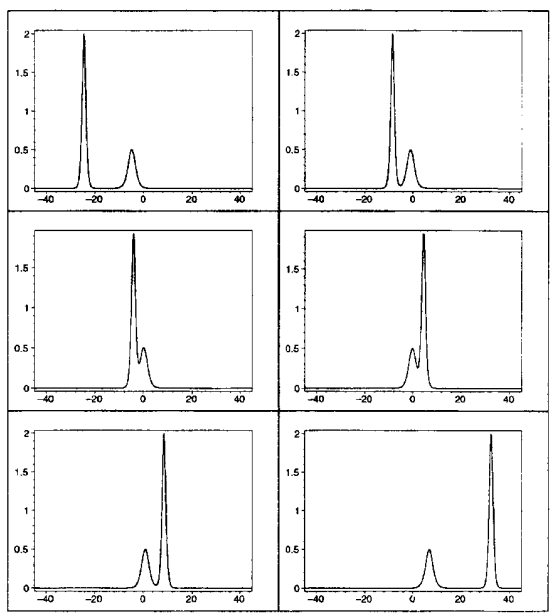

Figure 9. Movement, collision, and propagation of undeformed and undamped solitons. After Ref. [81].

We reiterate then that the relevant point to be stressed is that Fröhlich effect and Davydov soliton are phenomena arising out of the same nonlinear kinetic effects that are present in Eq. (4) for the populations $\nu_{\vec{q}}(t)$, and in Eq. (8) for the reciprocal lifetimes $\Gamma_{\vec{q}}$. We stress once again that as a consequence of the fact that, because of Fröhlich effect, the population of the modes lowest in frequency largely increase, concomitantly their lifetime also largely increases (i.e. their reciprocal lifetime $\Gamma_{\vec{q}}$ in Eq. (8) largely decreases), while for the modes at intermediate to high frequencies their lifetime largely decreases (their reciprocal lifetime $\Gamma_{\vec{q}}$ largely increases), as already illustrated in Fig. 8 [53]. Hence, in the expression for the average field amplitude of Eq. (12), after a fraction of picosecond following the application of the exciting pulse has elapsed, there survive for a long time the contributions from the modes lowest in frequency, a survival time that keeps increasing as the intensity $S$ increases. This implies that it may be expected that an excitation composed by a coherent interplay of the low-lying-in frequency excited polar (optical) modes in biopolymers, may propagate in the form of a Davydov solitary wave traveling undeformed and nearly undamped while Fröhlich's condensate state is maintained.

\section{Fröhlich's Effect in Non-polar Modes}

So far we have considered propagation of vibronic waves in biological media, but restricted to the case of polar modes. We briefly consider next the case of longitudinal acoustic modes. For that purpose we return to Eqs. (4), where now we take into account that the dispersion relation $\omega_{\vec{q}}$ is, for acoustic-like vibrations, $s q$, where $s$ is the velocity of sound in the media, this meaning that we are using a Debye model. Using this dispersion relation, and the Ansatz that the excitation is expected to be a closed-packet solitary wave, we arrive at the equivalent of Eq. (16), in this case acquiring the expression

$$
\begin{gathered}
i \frac{\partial}{\partial t} \psi(x, t)+\frac{\hbar}{2 M_{s}} \frac{\partial^{2}}{\partial x^{2}} \psi(x, t)+i \gamma_{s} \psi(x, t) \\
-G_{s}|\psi(x, t)|^{2} \psi(x, t)=0
\end{gathered}
$$

as shown in [54], with $M_{S}$ being a parameter with dimensions of mass. Evidently, this Eq. (21) is formally identical with Eq. (16) if in the latter we take $\omega_{0}=0$ and, of course with the coefficients being those corresponding to this case of LA (longitudinal acoustic) vibrations. However, a remarkable difference may be noticed, namely, while in Eq. (15) the coefficient in front of the second derivative in space is determined, through $\alpha$, by the bandwidth of the LO (longitudinal optical) vibrations dispersion relation, in this case, as shown in Ref. [54], it depends through the pseudo-mass $M_{s}$ on the characteristics of the experiment, that is, depends on the width of the solitary wave packet which is determined by the initial condition. The solution for a given hyperbolic secant-profile signal impinged on the system, say, the same as in the previous subsection, is given by

$$
\begin{gathered}
\psi(x, t)=\mathcal{A} \exp \left\{i\left[\frac{M_{s} v}{\hbar} x-\left(\omega_{s}-i \gamma_{s}\right) t-\frac{\theta}{2}\right]\right\} \\
\operatorname{sech}\left\{\mathcal{A}\left[\frac{\left|G_{s}\right| M_{s}}{\hbar}\right]^{1 / 2}(x-v t)\right\}
\end{gathered}
$$

where

$$
\omega_{s}=\frac{\left|G_{s}\right| \mathcal{A}^{2}}{2}-\frac{M_{s} v^{2}}{4 \hbar},
$$




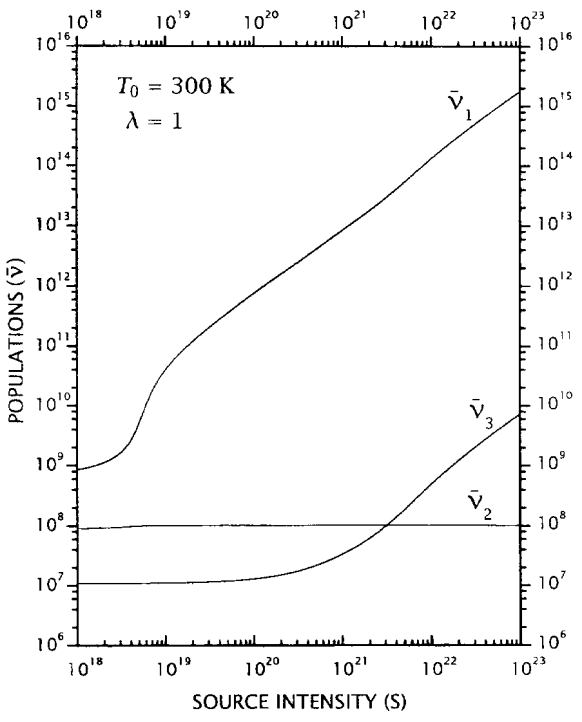

Figure 10. Acoustic vibrations: The steady state populations of the three relevant modes in the set — as described in the main text with increasing values of the intensity of the external source pumping modes labeled 2 and 3 in the ultrasonic region (after Ref. [54]).

As in the case of LO vibrations, the "acoustic" solitary wave is damped, and one may wonder if, as in the case of the "optical" solitary wave, this lifetime may be largely extended by the action of nonlinear kinetic terms enhanced by the pumping of energy on the system. We reconsider Eqs. (4), now specialized for the LA vibrations, and look for the stationary states when a constant exciting source is continuously applied [54].

To perform numerical calculations we choose a set of parameters in a typical order of magnitude approximation. We take for the Brillouin end-zone wavenumber $q_{B}=3.14 \times 10^{7} \mathrm{~cm}^{-1}$, $\omega_{\vec{q}}=s q \quad$ with $s=1.8 \times 10^{5} \mathrm{~cm} \mathrm{~s}^{-1}, \quad \Omega_{\vec{q}}=s_{B} q \quad$ with $s_{B}=1.4 \times 10^{5} \mathrm{~cm} \mathrm{~s}^{-1}$ (that is, Debye frequencydispersion relations for both the system, $\omega_{\vec{q}}$, and the bath, $\Omega_{\vec{q}}$ ). Moreover, the matrix elements $V^{(1)}$ and $V^{(2)}$ are proportional to the square roots of the wavenumbers [80], say $V_{\vec{q} \vec{q}^{\prime}}^{(1,2)}=K^{(1,2)}\left[\left|\vec{q}_{1} \| \vec{q}_{2}\right|\left|\vec{q}_{1}-\vec{q}_{2}\right|\right]^{\frac{1}{2}}$, and $K^{(1)}$ is determined from a typical value of $10 \mathrm{ps}$ for the lifetime $\tau_{\vec{q}}$ in Eq. (5) (for any system it can be determined from the linewidth in Raman scattering experiments). An open parameter $\lambda=\left|K^{(2)} / K^{(1)}\right|^{2}$ is introduced, and finally, $L$, the length of the sample in the direction of propagation is taken as $10 \mathrm{~cm}$. Therefore, the permitted wavenumbers for propagation of vibrations are contained in the interval $\pi / L \leq q \leq q_{B}$. For these characteristic values it follows that, because of energy and momentum conservation in the scattering events, the set of equations of evolution, Eqs. (4), which in principle couple all modes among themselves, can be separated into independent sets each one having nine modes. For example, taking the mode with the lowest wavenumber $\pi / L$, the set to which it belongs contains the modes $\kappa^{n-1} \pi / L$, where $\kappa=\left(s+s_{B}\right) /\left(s-s_{B}\right)=8$ in this case, and $n=2,3, \ldots, 9$. Let us call $\nu_{1}, \ldots, \nu_{9}$ the corresponding populations, having frequencies $\omega_{1}=5.6 \times 10^{4} \mathrm{~Hz}$, $\omega_{2}=4.5 \times 10^{5} \mathrm{~Hz} . \omega_{3}=3.6 \times 10^{6} \mathrm{~Hz}, \omega_{4}=2.9 \times 10^{7} \mathrm{~Hz}$,

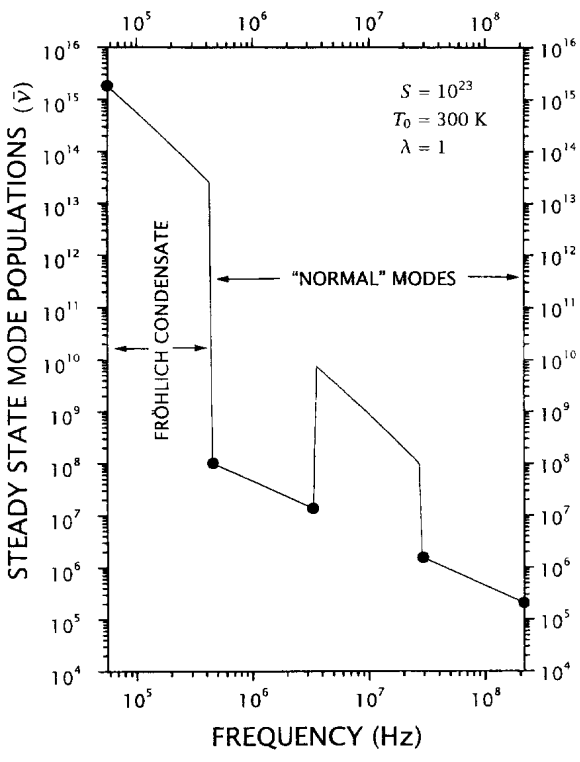

Figure 11. Acoustic vibrations: The population in the steady state for a pumping intensity $S=10^{23}$, of the modes along the spectrum of frequencies of the acoustic modes. Dots indicate the modes in the first set (the remaining part of the spectrum up to the highest Brillouin frequency $\omega_{B}=9.5 \times 10^{11} \mathrm{~Hz}$ has been omitted). (After Ref. [54]).

$\omega_{5}=2.3 \times 10^{8} \mathrm{~Hz}, \omega_{6}=1.8 \times 10^{9} \mathrm{~Hz}, \omega_{7}=1.5 \times 10^{10} \mathrm{~Hz}$, $\omega_{8}=1.2 \times 10^{11} \mathrm{~Hz}, \omega_{9}=9.5 \times 10^{11} \mathrm{~Hz}$. Moreover, for illustration, the open parameter $\lambda$ is taken equal to 1 , and we consider that only the modes 2 and 3 (in the ultrasonic region) are pumped with the same constant intensity $S=I \bar{\tau}$, where $I_{2}=I_{3}=I$, and $I_{1}$ and $I_{n}$ with $n=4, \ldots, 9$ are null, and $\bar{\tau}$ is a characteristic time used for scaling purposes (as in [29]) here equal to $0.17 \mathrm{~s}$. The results are shown in Fig. 10, where it is evident the large enhancement of the population in the mode lowest in frequency $\left(\nu_{1}\right)$, for $S_{0} \simeq 10^{19}$, at the expenses of the two pumped modes $\nu_{2}$ and $\nu_{3}$, while the modes $\nu_{4}$ to $\nu_{9}$ (higher in frequency) are practically unaltered.

The emergence of Fröhlich effect is clearly evidenced for this case of acoustical vibrations: In fact, pumping of the modes in a restricted ultrasonic band (in the present case in the interval $4.5 \times 10^{5} \mathrm{~Hz} \leq \omega \leq 2.8 \times 10^{7} \mathrm{~Hz}$ ), leads at sufficiently high intensity of excitation to the transmission of the pumped energy in these modes to those with lower frequencies $\left(\omega<\omega_{2}\right)$, while those with larger frequencies $\left(\omega>2.8 \times 10^{7} \mathrm{~Hz}\right)$ remain in near equilibrium, as shown in Fig. 11. It may be noticed that for the given value of $\bar{\tau}$, for $S=10^{23}$, the flux power provided by the external source, in the given interval of ultrasound frequencies being excited, is of the order of milliwatts. Modes in the interval $5.6 \times 10^{4} \leq \omega_{\vec{q}} \leq 4.5 \times 10^{5}$, those lowest in frequency, have large populations in comparison with those higher in frequency.

We can see then that there follows a close analogy with the case of polar vibrations, which is reinforced by the fact that the lifetime of the modes in the condensate also largely 


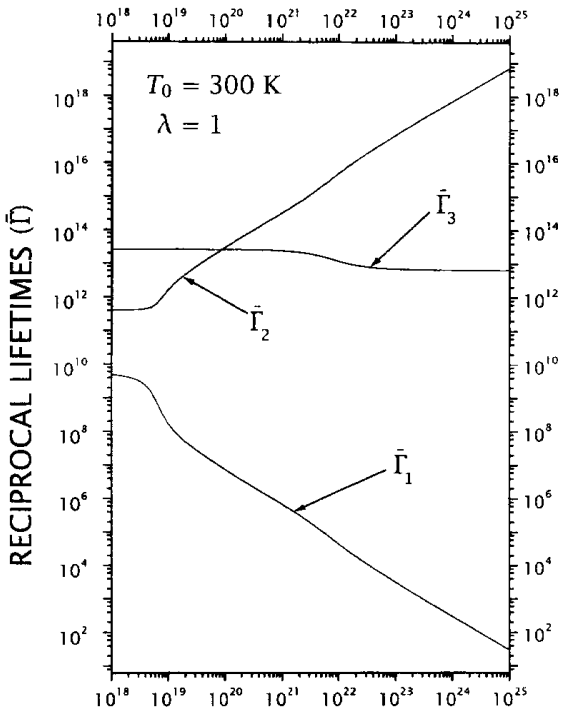

SOURCE INTENSITY $S$

Figure 12. Acoustic vibrations: The lifetime of the same modes as in Fig. 11, in terms of the intensity of the pumping source (after [54]).

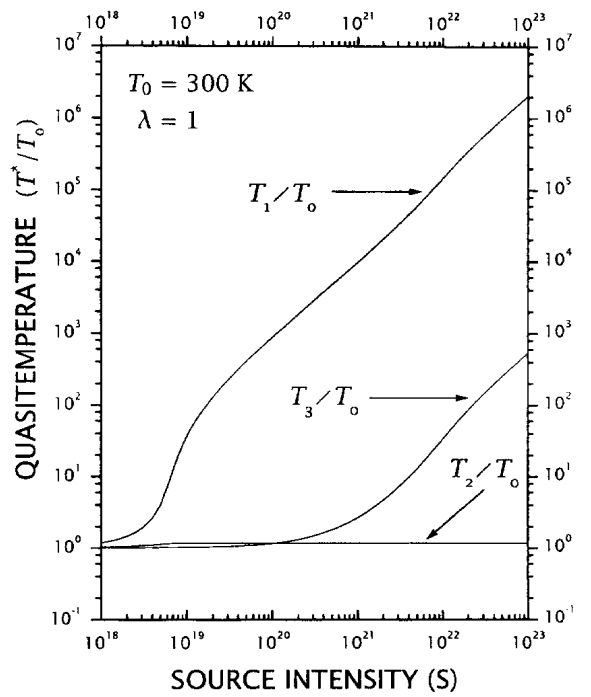

Figure 13. The quasi-temperature, defined in Eq. (36) for the modes in Fig. 10.

increases, as shown in Fig. 12 where the reciprocal of that lifetimes are displayed for the modes considered in Fig. 10. Therefore, the acoustical-vibrational soliton, composed by the coherent interplay of low-frequency acoustical modes, travels nearly undamped in the Fröhlich's condensate.

Moreover, we can also associate to the excited acoustical modes in Fig. 10 a quasitemperature as shown in Fig. 13, and quasi-chemical potentials in Fig. 14 - as defined by Eqs. (10) and (11) — which display the same behavior as in the case of polar vibrations.

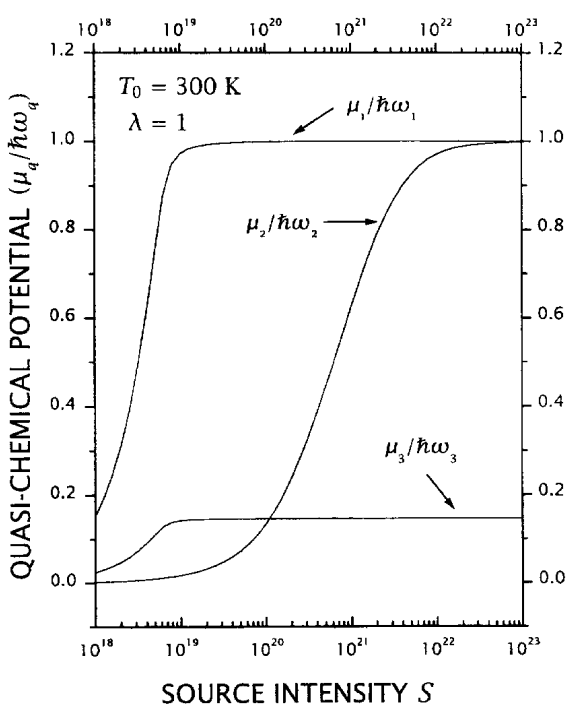

Figure 14. The quasi-chemical potential of the modes labeled 1 to 3 in Fig. 11, with mode 1 corresponding to the one with the lowest frequency in the given set: it is evident the emergence of a "BoseEinstein-like condensation" for $S$ approaching a critical value of the order of $10^{19}$.

\section{Fröhlich-Cherenkov Effect}

Considering either an "optical" or an "acoustical" soliton of the Davydov type respectively described in the previous sections, we recall that the amplitude and the velocity of propagation are determined by the initial condition of excitation (that is, the energy and the momentum transferred in the process of interaction with the external source). For example, in the case of the acetanilide we have considered in section 4, and in the conditions of the experiment of Careri et al. [72], the velocity of propagation is larger than the group velocity of the phonons in the optical branch corresponding to the CO-stretching vibrations, which is small because the dispersion relation is flat.

When the soliton velocity of propagation, say $v$, is larger than the group velocity of the normal vibrations, (the velocity of sound $s$ when the acoustic modes are involved), it may follow a Cherenkov-like effect. We recall that originally it was observed in connection with electromagnetic radiation by Cherenkov in 1934 [69]. It is a result that in a material media with an index of refraction $n$, the velocity of propagation of light is $c / n$, smaller than the velocity $c$ in vacuum (since $n>1$ ), and if an electron with velocity $u>c / n$ (but with the relativistic limitation of $u<c$ ) travels in this medium then, along a cone defined by the angle $\cos \theta=c / n u$, is emitted the so-called Cherenkov radiation: that is, along such direction photons are strongly emitted. This is the so-called superluminal radiation [82, 83].

Something similar is present in the case of phonons in the photoinjected plasma in semiconductors in the presence of an electric field: when the drift velocity $v$ of the carriers exceeds the group velocity of the $\vec{q}$-mode optical phonon, then along a cone whose axis is along the electric field, and 
with an aperture with angle $\theta_{\vec{q}}$ defined by

$$
\cos \theta=\omega_{\vec{q}} / v q,
$$

there follows a large emission of $\vec{q}$-mode optical phonons [84].

This is also the case when the soliton, either optical or acoustical, travels in bulk with a velocity $v$ larger than the group velocity of the normal vibronic waves. This is described elsewhere [54], and next we briefly outline the results. Inspection of Eq. (5) tells us that the presence of the direct coupling to the external source via $I_{\vec{q}}$ tends to increase the population of phonons. But, as already noticed, because of Fröhlich effect, such pumped energy tends to concentrate in the modes lowest in frequency, those at the Brillouin zone boundary in the case of optical vibrations and around the zone center in the case of acoustic vibrations.

Take the case of acoustic phonons, when there should be a large increase in the population of the modes with very small wavenumber. A straightforward calculation of Eq. (2a) leads to the result that (cf. Eq. (9) for LO phonons)

$$
\nu_{\vec{q}}(t)=\left[\mathrm{e}^{\mathrm{F}_{\tilde{\mathrm{q}}}(\mathrm{t})}-1\right]^{-1}+\left|\frac{\mathrm{f}_{\tilde{\mathrm{q}}}(\mathrm{t})}{\mathrm{F}_{\tilde{\mathrm{q}}}}\right|^{2} .
$$

Evidently, in the absence of the perturbation, that is, $I_{\vec{q}}=0$ and $\left\langle a_{\vec{q}}\right\rangle=0$ and then $f_{\vec{q}}=0$, it follows that $F_{\vec{q}}(t)=\hbar s q / k_{B} T_{0}$, and we recover the usual Planck distribution in equilibrium. In the presence of the perturbation we need to obtain both $F_{\vec{q}}(t)$ and $f_{\vec{q}}(t)$. On the one hand, a direct calculation tell us that

$$
\begin{gathered}
\left|f_{\vec{q}}(t) / F_{\vec{q}}(t)\right|^{2}=\left|\left\langle a_{\vec{q}} \mid t\right\rangle\right|^{2} \\
\approx \int_{0}^{L} \frac{d x}{L}|\psi(x, t)|^{2} \approx\left(\mathcal{A}^{2} w^{2} / L^{2}\right) \mathrm{e}^{-\gamma_{\mathrm{s}} \mathrm{t}},
\end{gathered}
$$

after using Eqs. (20) and (22) [54], and, we recall, under a sufficiently intense excitation $\gamma_{s}$ is small and then

$\left|<a_{q}\right| t>\left.\right|^{2}$ becomes near time independent; we have called $w$ the width of the solitary wave packet.

On the other hand, $F_{\vec{q}}$ in steady state conditions after application of the constant external excitation, depends on the intensity of the pumping source. We recall that this Lagrange multiplier may be rewritten in either of two alternative forms [cf. Eqs. (10) and (11)], which specifically in this case of steady-state acoustic vibrations in Debye model one is

$$
F_{\vec{q}}=\left[\hbar s q-\mu_{\vec{q}}\right] / k_{B} T_{0},
$$

where $\mu_{\vec{q}}$ plays the role of a quasi-chemical potential for mode $\vec{q}[85,66]$ as we have already noticed in the Section on polar vibrations. In Fig. 14 is shown the dependence of the quasi-chemical potentials, corresponding to the modes in Fig. 10, with the pumping intensity. The other is

$$
F_{\vec{q}}=\hbar s q / k_{B} T_{\vec{q}}^{*},
$$

introducing the quasitemperature $T^{*}$ per mode, as it is done in semiconductor physics [86]. In Fig. 13 is shown the dependence of the quasitemperature, corresponding to the modes in Fig. 10, with the pumping intensity.

Let us take the choice of Eq. (28), then the quasitemperature $T_{\vec{q}}^{*}$ is given by

$$
k_{B} T_{\vec{q}}^{*}=\hbar s q / \ln \left[1+\frac{1}{\nu_{\vec{q}}-\left|\left\langle a_{\vec{q}}\right\rangle\right|^{2}}\right]
$$

and we recall that

$$
\left|\left\langle a_{\vec{q}}\right\rangle\right|^{2} \approx \mathcal{A}^{2} w^{2} / L^{2},
$$

(for $\gamma_{s} \rightarrow 0$, with $\nu_{\vec{q}}$ determined in each case solving Eq. (4)). Using Eqs. (27) to (30) we have that [54]

$$
\mu_{\vec{q}}=\hbar v q \cos \eta_{\vec{q}}
$$

where

$$
\cos \eta_{\vec{q}}=\frac{s}{v}\left[1-\frac{k_{B} T_{0}}{\hbar s q} \ln \left[1+\left(\nu_{\vec{q}}-\left|\left\langle a_{\vec{q}}\right\rangle\right|^{2}\right)^{-1}\right]\right]=\frac{s}{v}\left[1-\frac{T_{0}}{T_{\vec{q}}^{*}}\right]
$$

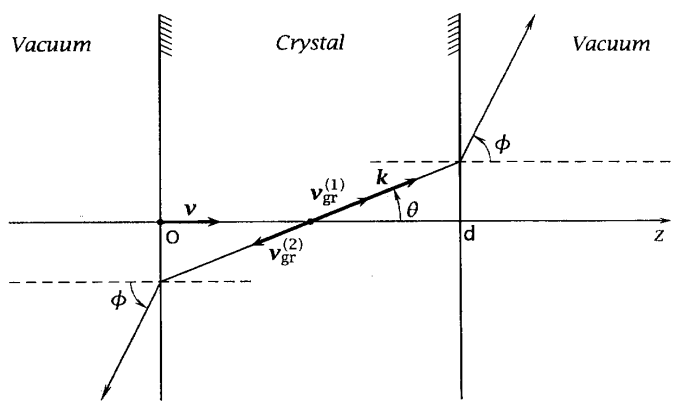

Figure 15. The direction of propagation of the waves of Cherenkov radiation, when spatial dispersion is taken into account, for the ordinary wave (subscript 1) and the anomalous wave (subscript 2) (Adapted from Ref. [83])
These results imply in this case in a phenomenon of a peculiar character which we call Fröhlich-Cherenkov-effect. In fact, we note, first, that there follows a large enhancement of phonons in mode $\vec{q}$ for $\mu_{\vec{q}}$ approaching $\hbar s q$, and second, the linear motion of the soliton defines a particular direction, the one given by its velocity of propagation $\vec{v}$. Therefore, there is a preferential direction of production of vibrational waves determined by the condition

$$
\hbar s q \quad \approx \hbar v q \cos \eta_{\vec{q}}=\mu_{\vec{q}}
$$

what implies in that

$$
\cos \eta_{\vec{q}} \quad \approx s / v
$$


Equation (33) defines the direction of propagation $\vec{q}$ of the longitudinal vibration and its modulus. Since $\eta_{\vec{q}}$ depends

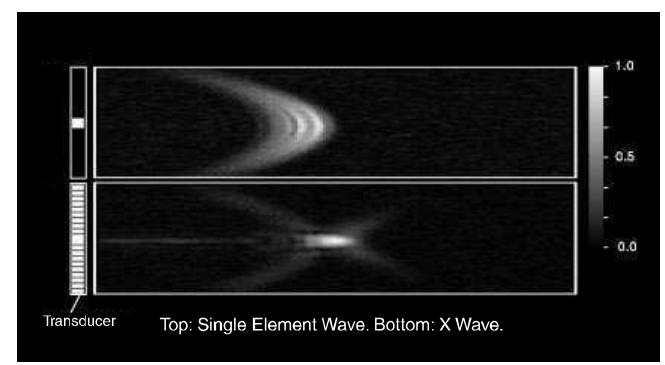

Figure 16. Normal sound propagation (upper figure), and the excitation interpreted as a supersonic soliton (lower figure); from Ref. [88] (We thank W. A. Rodrigues and J. E. Maiorino for providing us with a postscript file of this picture).

only on the modulus of $\vec{q}$, there follow two Cherenkov-like privileged directions of emission of $\vec{q}$-mode phonons, one forward and one backwards, like the normal and anomalous Cherenkov cones in radiation theory as illustrated in Fig. 15 adapted from [83]. In the present case both directions are symmetrical on both sides of the centre defined at each time by the position occupied by the soliton. This may account for the observed so-called X-waves [35, 87, 88]. In Fig. 16 is illustrated the cases of propagation of the normal sound wave (upper figure) and of the, presumably, solitary wave-packet selectively excited by the transducer (lower figure) with velocity larger than the sound velocity in the medium. The figure has appeared in [88]. Given the angle $\eta_{\vec{q}}$ (called the axicon angle in [35]), then $v$ is larger than $s$ in the percentage $\left[\left(v / \cos \eta_{\vec{q}}\right)-s\right] / v$. Same arguments are valid for the case of the optical soliton, when $\nabla_{\vec{q}} \omega_{\vec{q}}$ (the group velocity of the normal mode) enters in place of $s$. In the case of Fig. 16, a rough estimate gives $\eta \sim 13^{\circ}$ and $v_{s} / s \sim 1.02$, that is, the velocity of propagation of the soliton, $v$, is roughly $2 \%$ larger than the velocity of sound in the medium.

\section{Evidencing Fröhlich's Effect}

In the Introduction we have already mentioned some instances of experiments which could be indicative of the existence of Fröhlich's effect. We here suggest two others, one of them in fact already performed, where the evidencing of Fröhlich's effect would be indirect, in the sense that what is observed is the Shrödinger-Davydov soliton wich is propagating in Fröhlich's condensate. In this case - as we have discussed previously — the solitary wave largely enhances its lifetime.

Consider the measurement of absorbance in acetanilide, see Fig. 6, where it is evidenced the absorption band corresponding to the soliton. It has a certain bandwidth corresponding to the inverse of its lifetime in equilibrium at a given temperature $T_{0}$. But according to the results already presented, in the presence of an external pumping source the lifetime of the soliton, propagating in Fröhlich's condensate, largely increases, and then, there would follow a stretching of the band width in the absorption spectrum. The band width would further and further decrease when the intensity

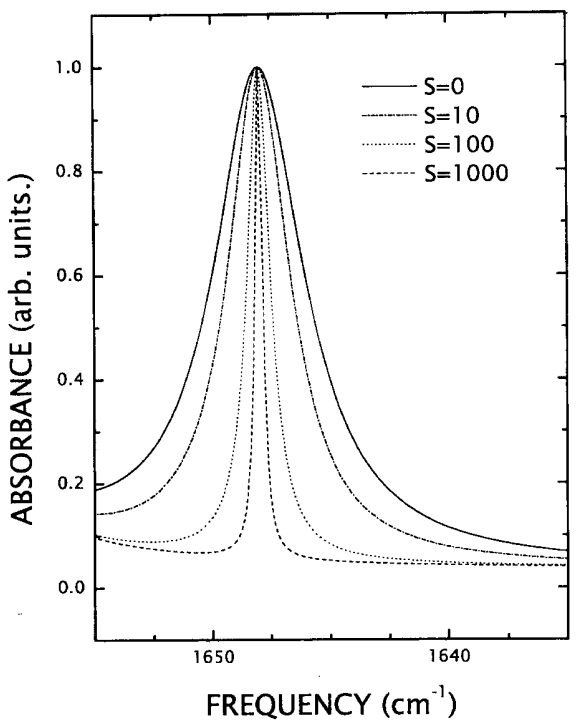

Figure 17. Modifications in the absorbance in acetanilide, with pumping of electromagnetic radiation in the infrared region corresponding to the vibrations of the $\mathrm{CO}$-stretching mode.

of the pumping source is augmented beyond the threshold for the onset of Fröhlich's effect.

If we consider the case of acetanilide excited by, for example, the pumping of electromagnetic radiation in the infrared region corresponding to the vibrations of the COstretching mode, it would be expected modifications, with increasing pumping intensity, as shown in Fig. 17.

But a case where this phenomenon seems to have already been evidenced is in the already mentioned experiments associated to the so-called "excitoner" $[36,37,38]$. In this case complex behavior occurs not in a system of vibrational modes, but in a system of boson-like particles as are the excitons in semiconductors.

Recently it has been evidenced the phenomenon of stimulated amplification of low energy exciton populations (SALEEP for short in what follows; the term 'excitoner' has been coined for this phenomenon on the basis of the resulting amplification of a cloud of coherent excitons using random excitons, much as a laser beam can be amplified with incoherent photons) [36, 37]. The experiment consists in that a laser beam pulse incident on the sample front produces a gas of excitons, a c.w. laser pumps energy on this photoinjected excitons, and a packet of them is detected on the other side of the platelet. We have presented [38] a theoretical analysis of the phenomenon on the basis of a nonequilibrium ensemble formalism for statistical thermodynamics, the one used in analyzing Fröhlich's effect and SchrödingerDavydov soliton in previous sections. The equations of evolution for the populations of excitons in the away-fromequilibrium conditions are derived. They consist in a set of coupled nonlinear integro-differential equations which show that the phenomenon SALEEP is a particular manifestation of Fröhlich effect.

We do not here go into the details of the formalism 
and the derivation of the kinetic equations, because there is a complete equivalence with those presented in [29] to which we refer the reader - , here only specifying the particular system under consideration. The Hamiltonian which describes this system is composed of the energy operator for the exciton gas, the lowest exciton state $(n=1)$ is considered and the exciton energy dispersion relation is $E_{1 \vec{k}}=-R_{y}^{x}+\hbar^{2} k^{2} / 2 M$, with $R_{y}^{x}$ being the excitonic Rydberg $\left(\simeq 100 \mathrm{meV}\right.$ in $\left.\mathrm{Cu}_{2} \mathrm{O}\right), \vec{k}$ runs over the Brillouin zone, $M$ is the mass of the exciton $\left(\simeq 3 m_{0}\right.$ in $\left.\mathrm{Cu}_{2} \mathrm{O}\right)$ and zero of energy is at the bottom of the conduction band. The exciton gas produced by the exciting ultrashort beam interacts with the lattice, and differently to the case of free carriers, the interaction with optical phonons can be disconsidered in comparison with the one associated with the acoustical phonons [89], the one we introduce: formally this contribution is the same as given in [29] once excitons enter in place of the polar vibrations. It is also included the interaction with the electromagnetic field of the c.w. laser and the one associated to spontaneous recombination effects (luminescense) [89]. Other interactions leading to relaxation effects are incorporated in the kinetic equations on a phenomenological basis. Once the Hamiltonian has been defined we need to introduce the statistical thermodynamic level of description, what we do along the same line as used for the study of vibrational modes in the previous sections. Hence, we introduce as basic variables the time-dependent exciton populations which we call $\nu_{\vec{k}}(t)$, and the energy of the acoustic phonons $\mathcal{E}_{B}(t)$
( $B$ for bath) with these phonons assumed to constantly remain in equilibrium with an external reservoir at temperature $T_{0}$ (2 $\mathrm{K}$ in the experiment of Ref. [37]), and then $\mathcal{E}_{B}$ is time independent. The accompanying intensive nonequilibrium thermodynamic variables (Lagrange multipliers in the variational approach to MaxEnt-NESOM) are designated by $F_{\vec{k}}(t)$ and $\beta_{0}=\left(k_{B} T_{0}\right)^{-1}$ respectively, the latter being the reciprocal of the reservoir temperature since the thermal bath is described by a canonical distribution in equilibrium. Variables $\nu$ and $F$ are connected, once the corresponding calculations in MaxEnt-NESOM are performed, by

$$
\nu_{\vec{k}}(t)=\left[\mathrm{e}^{\mathrm{F}_{\tilde{\mathrm{k}}}(\mathrm{t})}-1\right]^{-1},
$$

and one can choose [cf. Eq. (11)] to write

$$
F_{\vec{k}}(t)=E_{1 \vec{k}} / k_{B} T_{\vec{k}}^{*}(t),
$$

introducing the so-called quasi-temperature $T_{\vec{k}}^{*}$ per level, or, other choice can be [cf. Eq. (16)]

$$
F_{\vec{k}}(t)=\left[E_{1 \vec{k}}-\mu_{\vec{k}}(t)\right] / k_{B} T_{0},
$$

introducing a quasi-chemical potential $\mu_{\vec{k}}(t)$ per level as proposed, for example, by H. Fröhlich [90] and P. T. Landsberg [66], in a quite similar way to the one done in the case of the lattice vibrations in the previous sections.

The equations of evolution for the mesoscopic variables $\nu_{\vec{k}}(t)$ are calculated in the MaxEnt-NESOM-based kinetic theory. They are quite similar to those in [29], given by

$$
\begin{aligned}
\frac{d \nu_{\vec{k}}(t)}{d t}= & I_{\vec{k}}-\frac{\nu_{\vec{k}}(t)}{\tau_{\vec{k} R}}-\frac{\nu_{\vec{k}}(t)}{\tau_{\vec{k} D}}-\frac{\nu_{\vec{k}}(t)}{\tau_{\vec{k}}} \\
& +\frac{8 \pi}{\hbar^{2}} \sum_{\vec{k}^{\prime}}\left|V_{\vec{k} \vec{k}^{\prime}}^{(2)}\right|^{2}\left[\nu_{\vec{k}-\vec{k}^{\prime}}^{B}\left(\nu_{\vec{k}^{\prime}}-\nu_{\vec{k}}\right)-\nu_{\vec{k}}\left(1+\nu_{\vec{k}^{\prime}}\right)\right] \delta\left(\Omega_{\vec{k}-\vec{k}^{\prime}}+E_{1 \vec{k}^{\prime}}-E_{1 \vec{k}}\right) \\
& +\frac{8 \pi}{\hbar^{2}} \sum_{\vec{k}^{\prime}}\left|V_{\vec{k} \vec{k}^{\prime}}^{(2)}\right|^{2}\left[\nu_{\vec{k}-\vec{k}^{\prime}}^{B}\left(\nu_{\vec{k}^{\prime}}-\nu_{\vec{k}}\right)+\nu_{\vec{k}^{\prime}}\left(1+\nu_{\vec{k}}\right)\right] \delta\left(\Omega_{\vec{k}-\vec{k}^{\prime}}-E_{1 \vec{k}^{\prime}}+E_{\vec{k}}\right) .
\end{aligned}
$$

The several contributions on the right of this set of equations of evolution which couple the populations of all the exciton states are: The first is the rate of production generated by the pumping c.w. laser; basically it consists of an absorption coefficient times the power of the incident radiation and we have called it $I_{\vec{k}}$. The second term accounts for recombination effects, with recombination time $\tau_{\vec{k} R}$ (of the order of $\mu \mathrm{s}$ in $\mathrm{Cu}_{2} \mathrm{O}$ ); the third accounts for diffusion, with diffusion time $\tau_{\vec{k} D}$, out of the initial volume where the excitons were produced (the region of focalization of the pumping-laser pulse [91]); the fourth incorporates scattering by impurities and all other mechanisms that may be present. It can be noticed that the three terms can be rewritten as $\nu_{\vec{k}} / \bar{\tau}_{\vec{k}}$, with $\bar{\tau}_{\vec{k}}$ then given by Mathiessen rule $\left(\bar{\tau}_{k}^{-1}=\tau_{\vec{k} R}^{-1}+\tau_{\vec{k} D}^{-1}+\tau_{\vec{k}}^{-1}\right)[92]$.
The fifth term is the contribution which is responsible for Fröhlich's effect [cf. Eq. (6)] in far-from-equilibrium boson systems, in the present case for SALEEP: In fact, in Eq. (37) after some calculus it can be evidenced that it contains a nonlinear term of the form

$$
\left(1-\mathrm{e}^{\beta_{0}\left(E_{1 \vec{k}}-E_{1 \vec{k}^{\prime}}\right)}\right) \nu_{\vec{k}}(t) \nu_{\vec{k}^{\prime}}(t),
$$

which for $E_{1 \vec{k}}<E_{1 \vec{k}^{\prime}}$ is a pumping term enhancing the population of state $|1, \vec{k}\rangle$ at the expenses of all others higher in energy. Reciprocally, for $E_{1 \vec{k}}>E_{1 \vec{k}^{\prime}}$ it is a relaxation term implying in a transfer of energy from state $|1, \vec{k}\rangle$ to states $\left|1, \vec{k}^{\prime}\right\rangle$. Consequently, we must expect the emergence of Fröhlich's effect, through a cascading-down process, leading to the result that the energy pumped on the system is concentrated in the modes lowest in energy, i.e. at the bot- 
tom of the exciton band.

We illustrate numerically these results using parameters characteristic of $\mathrm{Cu}_{2} \mathrm{O}$, and the conditions of the experiment

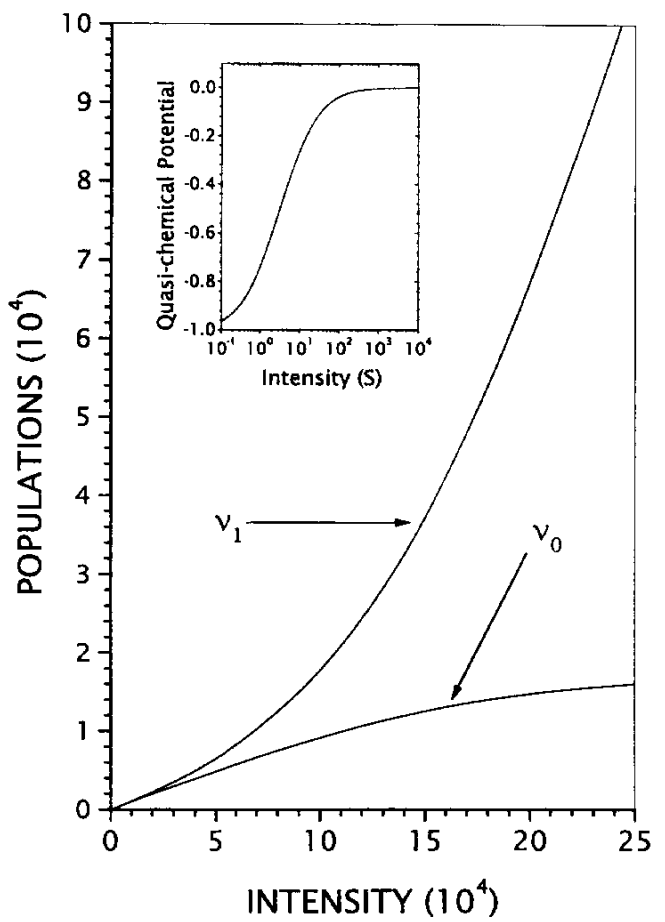

Figure 18. The populations in the steady state of a characteristic mode in Fröhlich condensate, $\nu_{1}$, and a "normal" mode, $\nu_{0}$, for intensities $S=I \bar{\tau}$. In the inset is shown the quasi-chemical potential $\mu_{1}^{x}=\mu_{1}-R_{y}^{x}$ of the mode in Fröhlich condensate, in units of $R_{y}^{x}$.

in Ref. [37]. Front sample is illuminated with a laser pulse with $\lambda=532 \mathrm{~nm}, 10 \mathrm{~ns}$ duration, and intensity $\approx 6.3$ MW $\mathrm{cm}^{-2}$; it is imposed illumination by a c.w. laser with $\lambda=605.4 \mathrm{~nm}$, and $4 \mathrm{~W} \mathrm{~cm}^{-2}$ of power flux (at this wavelength the absorption coefficient is roughly $\left.30 \mathrm{~cm}^{-1}\right)$. We also use for the mass of the exciton $M=3 m_{0}$, a static dielectric constant $\epsilon_{0}=10$ and the optical one $\epsilon_{\infty}=4$. Moreover, we consider a nearly wavevector-independent relaxation time $\bar{\tau}_{\vec{k}}(\equiv \bar{\tau})$ in Eq. (37) which is used as a scaling parameter defining a scaled time $\bar{t}=t / \bar{\tau}$. Noting that the set of equations of evolution for the populations couple all the exciton states over the Brillouin zone, they have been solved computationally however introducing approximations consisting in a partition of the Brillouin zone in a finite and small number of spherical layers, and taking a similar behavior of the population in each interval of involved states. This is sufficient to characterize the results, introducing only eventual numerical deviations but this can be improved when comparison with future detailed experimental data may be attempted. This done, we present the results in Figs. 18 and 19. Figure 18 shows the evolution in time of two characteristic populations in the steady state depending on the intensity $S=I \bar{\tau}$ of the source, which we take as the same for each exciton state. We recall that $I$ times the excitonic Rydberg is a fraction - determined by the absorption coefficient - of the intensity $4 \mathrm{~W} \mathrm{~cm}^{-2}$ of the pumping c.w. laser. The mode labelled with index one corresponds to a low-lying-in-energy exciton, and index zero for an exciton

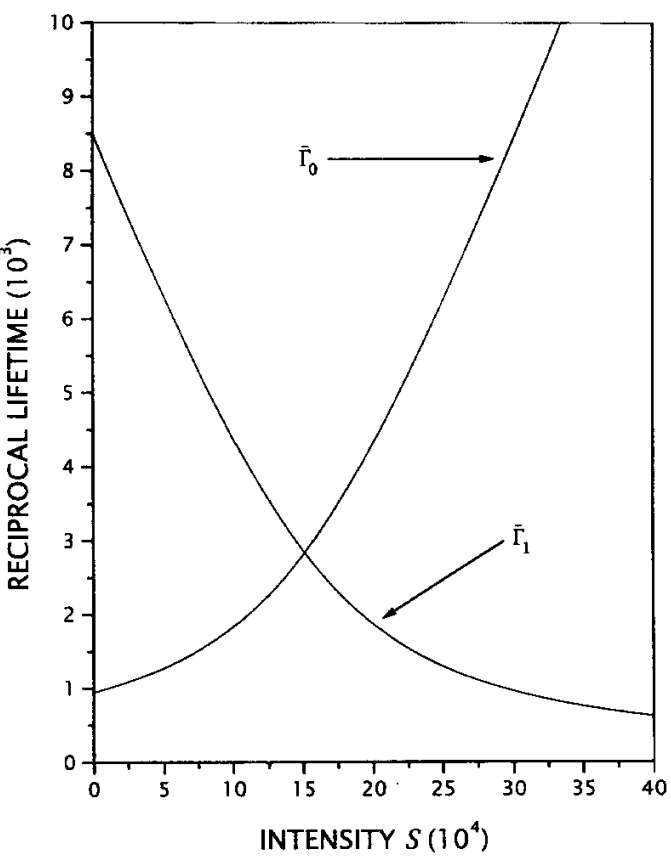

Figure 19. The reciprocal of the lifetimes of the modes of Fig. 1, where $\bar{\Gamma}_{1(0)}=\bar{\tau} / \tau_{1(0)}$.

higher in energy. In the inset is described the evolution of the quasi-chemical potential associated to $\nu_{1}$ [cf. Eq. (16) and (35b)], which we have written as $\mu_{1}^{x}=\mu_{1}-R_{y}^{x}$.

We once again stress that the quasi-chemical potential never coincides with the energy of the exciton state, i.e. $\mu_{0}^{x}$ is always negative and different from zero, but tends asymptotically to this value as the pumping intensity tends to infinity; hence a, say, strict Bose-Einstein-like condensation does not occur. What emerges is a large amplification of the populations over a certain region of the exciton states low in energy, constituting what we have already called Fröhlich condensate. It is worth noticing that also in this case we can see the presence of a kind of "two-fluid system": excitons in the Fröhlich condensate (a "superphase") and incoherents excitons in a "normal phase", similarly to the situation shown by Fig. 3 in the case of optical vibrations.

Inamura and collaborators [93] developed a simplified modelling of the system, keeping only the presence of what we have called Fröhlich term in the equation of evolution for the populations of excitons. We can then say that this phenomenon which is considered a kind of Bose-Einstein condensation of excitons is, under a proper look, a particular manifestation of the decades-old Fröhlich's effect, and therefore to its stydy can be applied all the results valid for the latter. In particular we call the attention to the two important associated novel phenomena previouly described, namely that the nonlinear terms present in the kinetic equations, Eq. (37), are responsible for a complex behavior in far-from-equilibrium boson systems consisting, besides of Fröhlich's effect, in the propagation of nearly undamped solitary waves of the Schrödinger-Davydov type. Here we recall the form of the reciprocal of the lifetime $\tau_{p \vec{k}}(t)$ of the modes composing the spatially constrained packet which is the soliton, namely, when given in units of $\bar{\tau}$, 


$$
\begin{aligned}
\frac{\bar{\tau}}{\tau_{p k}(t)} & =\frac{1}{2}+\frac{4 \pi \bar{\tau}}{\hbar^{2}} \sum_{\vec{k}^{\prime}}\left|V_{\vec{k} \vec{k}^{\prime}}^{(2)}\right|^{2}\left[1+\nu_{\vec{k}^{\prime}}+\nu_{\vec{k}-\vec{k}^{\prime}}^{B}\right] \delta\left(\Omega_{\vec{k}-\vec{k}^{\prime}}+E_{1 \vec{k}^{\prime}}-E_{1 \vec{k}}\right)+ \\
& -\frac{4 \pi \bar{\tau}}{\hbar^{2}} \sum_{\vec{k}^{\prime}}\left|V_{\vec{k} \vec{k}^{\prime}}^{(2)}\right|^{2}\left[\nu_{\vec{k}^{\prime}}-\nu_{\vec{k}-\vec{k}^{\prime}}^{B}\right] \delta\left(\Omega_{\vec{k}-\vec{k}^{\prime}}-E_{1 \vec{k}^{\prime}}+E_{1 \vec{k}}\right) .
\end{aligned}
$$

where $\Omega_{\vec{k}-\vec{k}^{\prime}}=s\left|\vec{k}-\vec{k}^{\prime}\right|$ is the dispersion relation of the acoustic phonons, and $\nu_{\vec{k}-\vec{k}^{\prime}}^{B}$ their distribution in equilibrium at temperature $T_{0}$.

On the basis of these results, we can arrive to the conclusion that the packet of excitons flowing ballistically from the condensate (in Ref. [37] with a velocity of roughly $4.5 \times$ $10^{5} \mathrm{~cm} / \mathrm{s}$ ) is an exciton-composed Schrödinger-Davydov soliton, something similar to what is the case in conducting polymers [48] when composed of carriers and in optical fibers [94] when composed of photons. This is reinforced by the fact that the amplitude of the signal, when the c.w. laser is present, is larger when compared with the one in its absence as reported in Ref. [37], what is consistent with the result that the lifetime of the soliton is increased with increasing levels of excitation as already discussed in connection with vibrations. Moreover, it can be shown that the profile of the signal in Ref. [37] is well fitted by a squared hyperbolic secant characteristic of Schrödinger-Davydov soliton as the one in Eq. (16), as shown in Fig. 20.

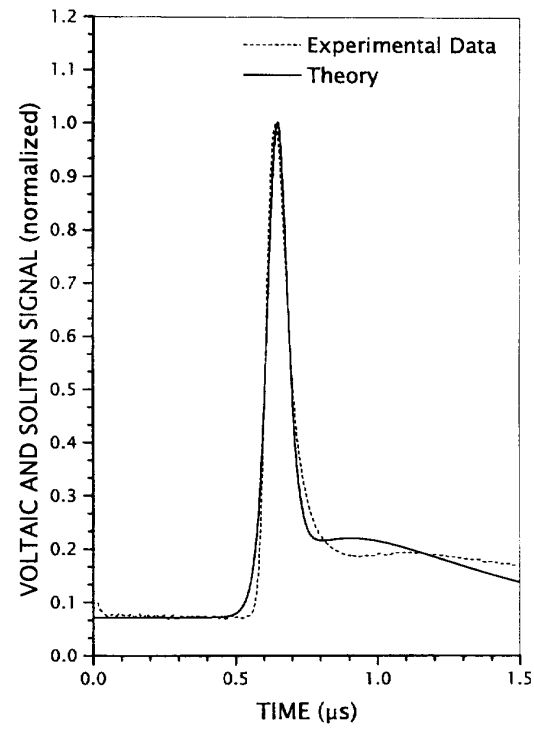

Figure 20. Comparison of the shape of the voltaic signal in Ref. [37] with the shape of the energy density of SchrödingerDavydov's soliton [proportional to the squared modulus of the amplitude field of Eq. (22)] plus the contribution of the incoherent excitons.

To draw the full line - the theoretical calculation - we have taken into account that, within the two fluid model, the registered signal is composed of the solitary wave formed by the coherent excitons plus the contribution of an accompanying cloud of incoherent excitons. The former, as noticed, consists of the squared modulus of the Schrödinger-
Davydov soliton-field amplitude of Eq. (40) below; the other is composed by the travelling normal excitons produced in the active region defined by the extinction length of the laser field, of the order of $0.033 \mathrm{~cm}$, and are decaying with a halftime of the order of $0.5 \mu \mathrm{s}$. These are the parameters used in the calculation, while the amplitude and width of the solitary wave have been fitted. The profile of the soliton field is

$$
\begin{gathered}
\psi(x, t)=\mathcal{A} \exp \left\{\mathrm{i}\left[\kappa x-\left(\omega_{s}-\mathrm{i} \gamma_{s}\right) t\right]-\theta / 2\right\} \\
\operatorname{sech}[W(\kappa x-v t)],
\end{gathered}
$$

where $\mathcal{A}$ is the amplitude; $\omega_{s}=\mathcal{A}^{2}\left|G_{s}\right| / 2-\kappa v / 4$ is the frequency; $v$ is the velocity of propagation; $\gamma_{s}$ is the nearly vanishing damping coefficient; $\kappa=M v / \hbar$ is the wavenumber; $W=\left[\mathcal{A}^{2} M|G| / \hbar\right]^{1 / 2}$, and $G=|G| \mathrm{e}^{\mathrm{i} \theta}$ is the nonlinear coupling strength, in complete analogy with the acousticphonon-composed solution discussed in the previous section. $\mathcal{A}$ and $v$ are determined by the energy and momentum transferred by the impinging exciting photons.

Using Eq. (40), and that $W v$ is of the order of the width of the signal which is roughly $20.8 \mu \mathrm{s}^{-1}$, and $\mathcal{A} \simeq 0.87$, the strength in the nonlinear terms responsible for Fröhlich and Davydov phenomena can be estimated to be $|G| \approx$ $2000 \mathrm{~s}^{-1}$. We stress that the just described behavior of the system follows for a weak nonlinear kinetic term coupling excitons and thermal bath; this coupling strength and the amplitude of the signal are the only open parameters fixed by best fitting.

In conclusion we may say that the so-called "excitoner" can provide, in the way just described a verification of the existence of Fröhlich's effect in boson systems governed by nonlinear kinetic laws.

\section{Informational and Thermodynamic Properties of Fröhlich's Effect}

In the context of Informational-Statistical Thermodynamics (IST) [25], the relevant nonequilibrium thermodynamic state function is the quasientropy, or the informationalstatistical entropy in Jaynes-Shannon sense, which more properly should be called the quantity of uncertainty of information. It is given in terms of the nonequilibrium statistical operator by

$$
\bar{S}(t)=-\operatorname{Tr}\left\{\varrho_{\varepsilon}(t) \wp_{\varepsilon}(t) \ln \varrho_{\varepsilon}(t)\right\},
$$

where $\wp_{\varepsilon}(t)$ is a time-dependent projection operator (it is determined by the nonequilibrium state of the system at any 
time $t$ ), defined in Ref. [57], which has the property that

$$
\wp_{\varepsilon}(t) \ln \varrho_{\varepsilon}(t)=\ln \bar{\varrho}(t, 0),
$$

and we recall that $\varrho_{\varepsilon}(t)$ is the systems' nonequilibrium statistical operator in Zubarev's approach, and $\bar{\varrho}$ is the auxiliary ("instantaneously frozen") statistical operator $[57,58]$. The projection operator above projects any dynamical quantity on, at any time $t$, the subspace of informational variables (or "relevant" subspace). Returning to the case of the system of Section 3, once we disregard the amplitudes in Eq. (4) we have that [65]

$$
\begin{gathered}
\bar{S}(t)=-\operatorname{Tr}\left\{\varrho_{\varepsilon}(t) \ln \bar{\varrho}(t, 0)\right\}=\phi(t) \\
+\sum_{q} F_{q}(t) \nu_{q}(t)+\beta_{0} E_{B},
\end{gathered}
$$

where

$$
\phi(t)=\ln \operatorname{Tr} \exp \left\{-\sum_{q} F_{q}(t) \hat{\nu}_{q}-\beta_{0} \hat{H}_{0 B}\right\} \equiv \ln \bar{Z}(t)
$$

is the logarithm of a nonequilibrium partition function which we have called $\bar{Z}(t)$.

It can be shown that this informational entropy has a particularly differentiated dependence on $\lambda$ only after the onset of Fröhlich effect, i.e. for, roughly, $\bar{I} \sim 10^{4}$, resulting [cf. Fig. 2] in that the informational entropy decreases for increasing values of $\lambda$ (which, we recall, measures the strength of the nonlinear contribution responsible for the complex behavior of the system): this can be interpreted as some kind of increase in order, or of increase of information as a result of the formation of Fröhlich condensate. To characterize this point, we introduce the order parameter

$$
\Delta(\lambda)=\frac{\bar{S}_{0}^{\mathrm{ss}}-\bar{S}_{\lambda}^{\mathrm{ss}}}{\bar{S}_{0}^{\mathrm{ss}}},
$$

where $\bar{S}_{0}^{\text {ss }}$ and $\bar{S}_{\lambda}^{\text {ss }}$ are the entropies in the steady-state for $\lambda=0$ and $\lambda \neq 0$, which is shown in Fig. 21, where it is evidenced the above mentioned characteristics.

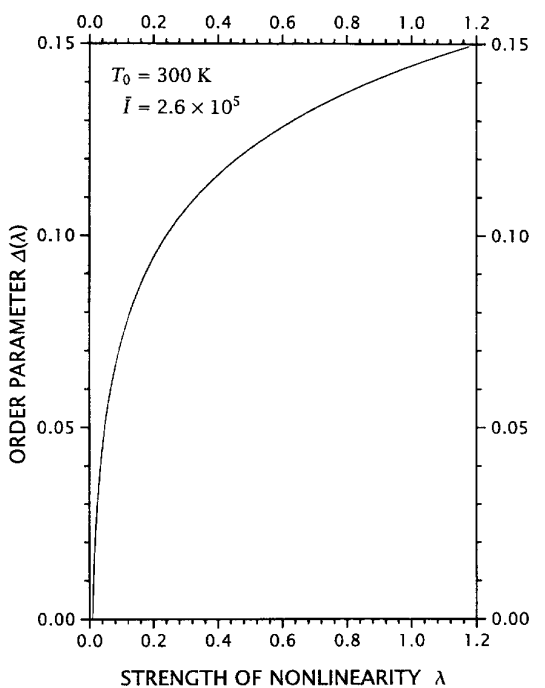

Figure 21. The order parameter of Eq. (45) as a function of the strength of the nonlinear coupling.
Next we consider the function informational-entropy production given by

$$
\begin{gathered}
\bar{\sigma}(t)=\frac{d}{d t} \bar{S}(t)=\sum_{q} F_{q}(t) \frac{d \nu_{q}(t)}{d t} \\
=\sum_{q} \frac{d \nu_{q}(t)}{d t} \ln \frac{\nu_{q}(t)+1}{\nu_{q}(t)},
\end{gathered}
$$

where we have used Eq. (25) but for $f_{\vec{q}}=0$, and the fact that $E_{B}$ does not change in time.

The production of informational entropy of Eq. (46), or IST-entropy production has two contributions:

$$
\bar{\sigma}(t)=\bar{\sigma}_{i}(t)+\bar{\sigma}_{e}(t)
$$

consisting of the so-called internal one, $\bar{\sigma}_{i}(t)$, which results from the internal interactions in the system, and the external one, $\bar{\sigma}_{e}(t)$, due to interactions with the surroundings, in this case with the source and the thermal reservoir. They are given by

$$
\begin{aligned}
& \bar{\sigma}_{i}(t)=\sum_{q}\left[F_{q}(t)-\beta_{0} \hbar \omega_{q}\right] \sum_{j=1}^{5} J_{\vec{q}_{(j)}}(t), \\
& \bar{\sigma}_{e}(t)=\sum_{q} F_{q}(t) I_{\vec{q}}^{(2)}(t)+\beta_{0} J_{T D}^{(2)}(t),
\end{aligned}
$$

where use was made of Eq. (4) [with $\zeta_{\vec{q}}=0$ ]: In Eq. (48) are present the contributions from the internal interactions of the open system and the bath, and of the latter with the former; we can see that $F_{\vec{q}}(t)-\beta_{0}=\left[k_{B} T_{\vec{q}}(t)\right]^{-1}-\left[k_{B} T_{0}\right]^{-1}$, which is zero only when all $T_{\vec{q}}$ are equal to $T_{0}$, i.e. when final equilibrium has been attained (the one that follows asymptotically after the source is switched off, that is, for $I_{\vec{q}}=0$ ). In Eq. (49) the first term on the right is the one due to the coupling of the open system and the source, and the second one accounts for the diffusion of heat from the bath to the reservoir (the latter has been assumed to be sufficiently rapid for keeping the thermal bath, which is receiving energy from the open system of polar vibrations, constantly in equilibrium with the reservoir at temperature $T_{0}$, as it has been constantly used in our analysis).

We omit the corresponding figures, and suffice it to say that $\bar{\sigma}_{i}(t)$ is definite positive, and at the steady state $\bar{\sigma}_{e}(t)=-\sigma_{i}(t)$ as it should since the total $\bar{\sigma}(t)$ is null. The positiveness of $\sigma_{i}(t)$ can be considered to be a manifestation of a kind of $\mathcal{H}$-theorem in MaxEnt-NESOM-based IST $[25,95]$. Moreover, during their evolution in time the internal and external IST-entropy production for non-null values of $\lambda$ (the nonlinear coupling strength) are, in modulus, ( $\bar{\sigma}_{i}$ is positive but $\bar{\sigma}_{e}$ is negative), larger than the one for $\lambda=0$ (absence of the nonlinear coupling). However, the total $\bar{\sigma}(t)=\bar{\sigma}_{i}(t)+\bar{\sigma}_{e}(t)$ is smaller for $\lambda \neq 0$ than for $\lambda=0$, what is reflected in the fact that the informational entropy for finite $\lambda$ is smaller than the one for $\lambda=0$, what is seen in Fig. 21 [cf. Eq. (45)], thus, as noticed, showing the increase in information created by the onset of Fröhlich condensation. 
We consider in continuation two additional important results in IST [25, 95], which are generalizations of those of Generalized Irreversible Thermodynamics [6, 10]. One is the criterion for evolution: the change in time of IST-entropy production can be separated out into two parts, namely

$$
\frac{d}{d t} \bar{\sigma}(t)=\frac{d_{F}}{d t} \bar{\sigma}(t)+\frac{d_{Q}}{d t} \bar{\sigma}(t)
$$

where

$$
\begin{aligned}
& \frac{d_{F}}{d t} \bar{\sigma}(t)=\sum_{q} \frac{d}{d t} F_{q}(t) \frac{d}{d t} \nu_{q}(t), \\
& \frac{d_{Q}}{d t} \bar{\sigma}(t)=\sum_{q} F_{q}(t) \frac{d^{2}}{d t^{2}} \nu_{q}(t),
\end{aligned}
$$

that is, the change in time of the informational-entropy production due to the change in time of the Lagrange multipliers, and the other due to the change in time of the basic variables (the populations), and we recall that $d E_{B} / d t=0$. After a simple calculation of Eq. (51) using Eq. (25) (with $f_{\vec{q}}=0$ ) we find that

$$
\frac{d_{F}}{d t} \bar{\sigma}(t)=-\sum_{q} \frac{1}{\nu_{q}(t)\left(\nu_{q}(t)+1\right)}\left(\frac{d}{d t} \nu_{q}(t)\right)^{2} \leq 0
$$

verifying for this system the generalization [25, 95] of Glansdorff-Prigogine's thermodynamic (originally called universal) criterion of evolution. That is, along the trajectory of the macrostate of the system in the thermodynamic (or Gibbs) space of states, the quantity of the Eq. (51) is always non-negative, a quantity which in classical Onsagerian thermodynamics is the product of the change in time of the thermodynamic forces times the fluxes of matter and energy $[6,10,95]$.

Finally, we look for the criterion for (in)stability, which requires the analysis of the quantity called the excess of entropy production. First we introduce the quantity

$$
\frac{1}{2} \delta^{2} \bar{S}(t)=\frac{1}{2} \sum_{q q^{\prime}}\left[\frac{\delta^{2} \bar{S}(t)}{\delta \nu_{q}(t) \delta \nu_{q^{\prime}}(t)}\right]^{\mathrm{ss}} \Delta \nu_{q}(t) \Delta \nu_{q^{\prime}}(t),
$$

where $\Delta \nu_{q}(t)$ represents the value of an imposed arbitrary deviation from the steady-state of the system $\left(\delta^{2} \bar{S}\right.$ is the second functional differential of the IST entropy). A direct calculation tells us that

$$
\frac{\delta^{2} \bar{S}(t)}{\delta \nu_{q}(t) \delta \nu_{q^{\prime}}(t)}=-\frac{\delta_{q q^{\prime}}}{\nu_{q}(t)\left(\nu_{q}(t)+1\right)},
$$

and consequently

$$
\frac{1}{2} \delta^{2} \bar{S}(t)=-\frac{1}{2} \sum_{q}\left[\frac{1}{\nu_{q}^{\mathrm{ss}}\left(\nu_{q}^{\mathrm{ss}}+1\right)}\right]\left|\Delta \nu_{q}(t)\right|^{2} \leq 0,
$$

what is a manifestation of the convexity of the maximized informational entropy. Differentiation in time of Eq. (56) introduces the quantity called excess of entropy production function, namely

$$
\begin{gathered}
\delta^{2} \bar{\sigma}(t)=\frac{1}{2} \frac{d}{d t} \delta^{2} \bar{S}(t)=-\sum_{q} \frac{\Delta \nu_{q}(t)}{\nu_{q}^{\mathrm{ss}}\left(\nu_{q}^{\mathrm{ss}}+1\right)} \frac{d}{d t} \Delta \nu_{q}(t) \\
=\sum_{q} \Delta F_{q}(t) \Delta \nu_{q}(t)
\end{gathered}
$$

a quantity shown in Fig. 22, where we can see that it is nonnegative, and therefore

$$
\frac{1}{2} \delta^{2} \bar{S}(t) \delta^{2} \bar{\sigma}(t) \leq 0
$$

is always non-positive and then, according to Lyapunov theorem in linear stability analysis (see for example [6]) the macroscopic state of this system displaying Fröhlich effect, is always stable. This is a manifestation of the generalization in IST [25, 95] of Glansdorff-Progogine's (in)stability criterion $[6,10]$.

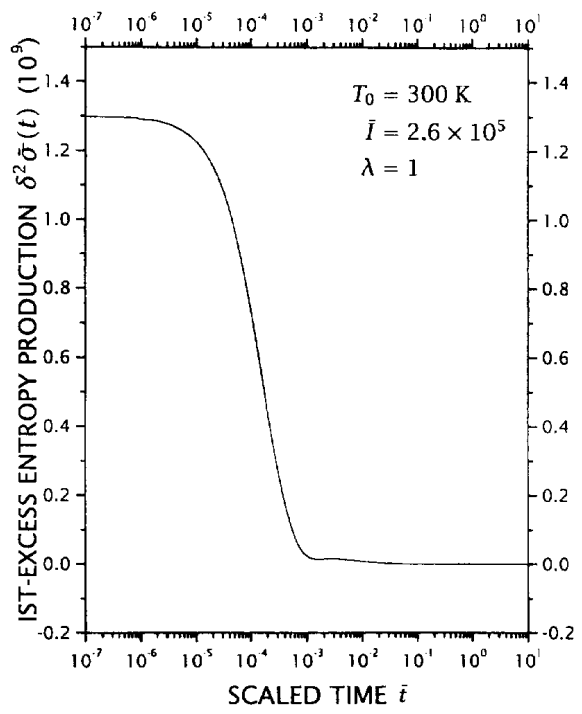

Figure 22 . The excess entropy production around the steady state.

The stability of the macroscopic state of the system can be characterized in an alternative way, consisting in that for the particular present case - it can be proved a generalization of Prigogine's theorem of minimum entropy production, which ensures the stability of any thermodynamic system in the immediate neighborhood of the state of equilibrium, i. e. within a strictly linear (or Onsagerian) regime of classical irreversible thermodynamics where Onsager's reciprocity relations are satisfied $[6,10]$. The theorem proves that in Onsager's strictly-linear regime and in the generalization to IST [25, 95], $\bar{\sigma}_{i} \geq 0$ and $d \bar{\sigma}_{i} / d t \leq 0$ in the immediate neighbourhood of the steady state are always satisfied and ensure the stability of the macrostate. In the model we are presenting it can be verified that in any condition, that is, even far away from equilibrium and then in the nonlinear regime outside Onsager's domain, the condition above is satisfied everywhere and then $\bar{\sigma}_{i}$ plays the role of a thermodynamic potential whose minimum defines an attractor for the steady states of the system. 


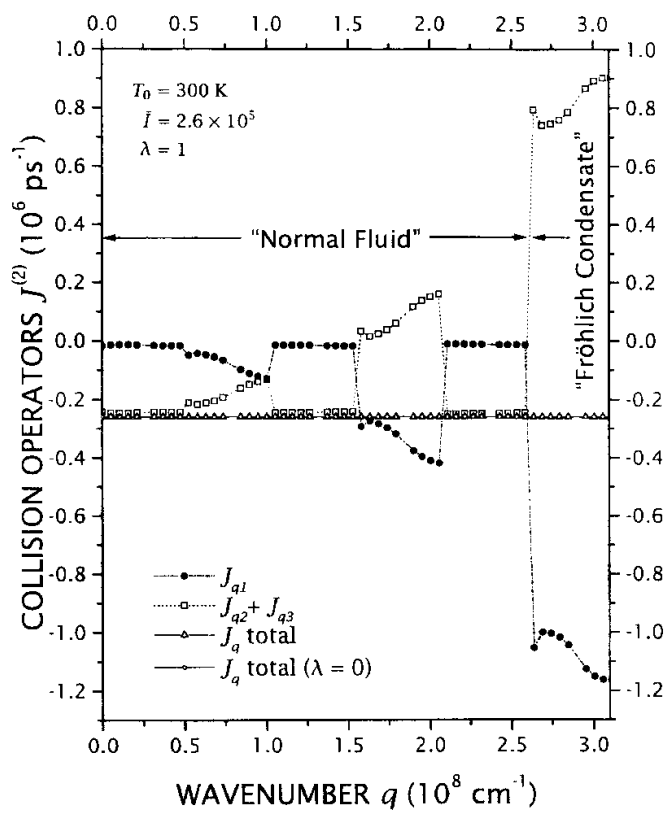

Figure 23. The different contributions to the relaxation processes for $\lambda=1$ and in the absence of a nonlinear coupling $(\lambda=0)$.

We recall that of the contributions $J_{q_{j}}^{(2)}$ in Eq. (4) the first two are purely responsible for relaxation processes (decay of energy from the polar system to the thermal bath through the anharmonic processes). The other contributions are responsible, as we have seen, for the transfer of energy between the modes lower in energy and those higher in energy (cf. in Eq. (6). This is better visualized in Fig. 23, where $J_{q_{1}}^{(2)}$ [standing for the contributions of Eq. (5)] is exclusively responsible for dissipation, while the other con- tributions (proportional to $\lambda$ and responsible for the emergence of Fröhlich's effect) produce only redistribution of energy among the modes: the lines with open circles, corresponding to the case with $\lambda=0$ (i.e. absence of nonlinearities), and with triangles, for $\lambda=1$, coincide. Moreover, for $q \gtrsim 2.6 \times 10^{8} \mathrm{~cm}^{-1}$ or $\omega \gtrsim 8.7 \times 10^{12} \mathrm{~Hz}$ (cf. Fig. 3), the contributions of $J_{q_{2}}$ plus $\widetilde{J}_{q_{3}}$ are, for $\lambda=1$, positive, that is, they act as a source term, the one in fact responsible for the "feeding" of the modes in the Fröhlich condensate, resulting in the large increase of their populations.

Furthermore, we are dealing with a nonlinear dissipative system and to it applies other relevant results in Nonlinear Science [96]. As Nicolis and Daems noticed [97] the equations of evolution [Eqs. (4) with $\zeta=0$ ] are nonlinear owing to the cooperativity inherent in the interactions. Moreover, the dissipative character of the set of equations is reflected in that when embedding the equations of evolution into the space of nonequilibrium thermodynamic states spawned by the set of variables of Eq. (1) (but omitting $a$ and $a^{\dagger}$ ) one has, on average, a contraction of a volume element each point of which follows the evolution laws. This very important property is shown to be equivalent in the case of Fröhlich condensation to

$$
\frac{1}{t-t_{0}} \int_{t_{0}}^{t} d t^{\prime} \sum_{\vec{q}} \frac{\partial}{\partial \nu_{\vec{q}}} \sum_{j=1}^{5} J_{q_{(j)}}\left(t^{\prime}\right)<0,
$$

where the sum is the so-called divergence of the evolution operator of Eqs. (4)[96], and the contribution associated to $E_{B}$ is absent because the assumption that the thermal bath remains constantly in equilibrium; hence $\partial\left[d E_{B} / d t\right] / \partial E_{B}$ (the divergence of its evolution operator) is null.

We have that

$$
\begin{aligned}
\frac{\partial}{\partial \nu_{\vec{q}}} \sum_{j=1}^{5} J_{\vec{q}_{j}}(t) & =-\frac{1}{2} \tau_{\vec{q}}^{-1}(t)-\frac{4 \pi}{\hbar^{2}} \sum_{\vec{q}^{\prime}}\left|V_{\vec{q} \vec{q}^{\prime}}^{(2)}\right|^{2}\left[1+\nu_{\vec{q}^{\prime}}(t)+\nu_{\vec{q}-\vec{q}^{\prime}}^{B}\right] \delta\left(\Omega_{\vec{q}-\vec{q}^{\prime}}+\omega_{\vec{q}^{\prime}}-\omega_{\vec{q}}\right) \\
& +\frac{4 \pi}{\hbar^{2}} \sum_{\vec{q}^{\prime}}\left|V_{\vec{q} \vec{q}^{\prime}}^{(2)}\right|^{2}\left[\nu_{\vec{q}^{\prime}}(t)-\nu_{\vec{q}-\vec{q}^{\prime}}^{B}\right] \delta\left(\Omega_{\vec{q}-\vec{q}^{\prime}}-\omega_{\vec{q}^{\prime}}+\omega_{\vec{q}}\right) \\
& -\frac{4 \pi}{\hbar^{2}} \sum_{\vec{q}^{\prime}}\left|V_{\vec{q} \vec{q}^{\prime}}^{(2)}\right|^{2}\left[\nu_{\vec{q}^{\prime}}(t)-\nu_{\vec{q}+\vec{q}^{\prime}}^{B}\right] \delta\left(\Omega_{\vec{q}+\vec{q}^{\prime}}-\omega_{\vec{q}^{\prime}}-\omega_{\vec{q}}\right) \equiv D_{q}(t) .
\end{aligned}
$$

These quantities $D_{q}(t)$ are predominantly negative for any $t$ (they are shown in Fig. 24 for a particular value of the intensity) and then Eq. (59) is verified.

Let us consider the Lyapunov exponents, $\lambda_{j}$, of the dynamical system when it has achieved the steady state, that is the eigenvalues of the linearized evolution operator, which are given by Eq. (60) once $\nu_{\vec{q}}$ is taken in the steady state. They are shown in Fig. 25 for a range of values of the intensity of the pumping source, once we use the results of Fig. 2. Inspection of this Fig. 25 tells us that the Lyapunov exponents are negative, what then ensures the stability of the solution for any value of the intensity, a point we have previously demonstrated through an alternative treatment. Other interesting point is that the modulus of the one corresponding to the mode labelled 1 (the one in Fröhlich condensate) is very small. This has the consequence that, since the population $\nu_{1}$ in the steady state can be shown to be proportional to the inverse of the modulus of $\lambda_{1}$, then it is the very large one corresponding to the mode in Fröhlich condensate. It can be noticed that the other quantities $\lambda_{3}$ and $\lambda_{5}$ also appear to be 
small, but whereas $\lambda_{1}$ approaches zero, the other two have values of hundred and thousand units since the scale is $10^{4}$.

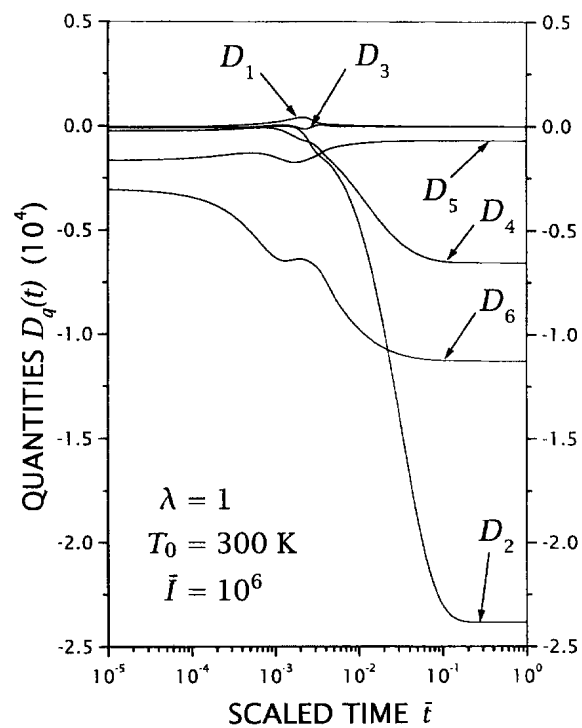

Figure 24. Quantities $D_{q}(t)$ of Eq. 60 for the set of modes of Fig. 2.

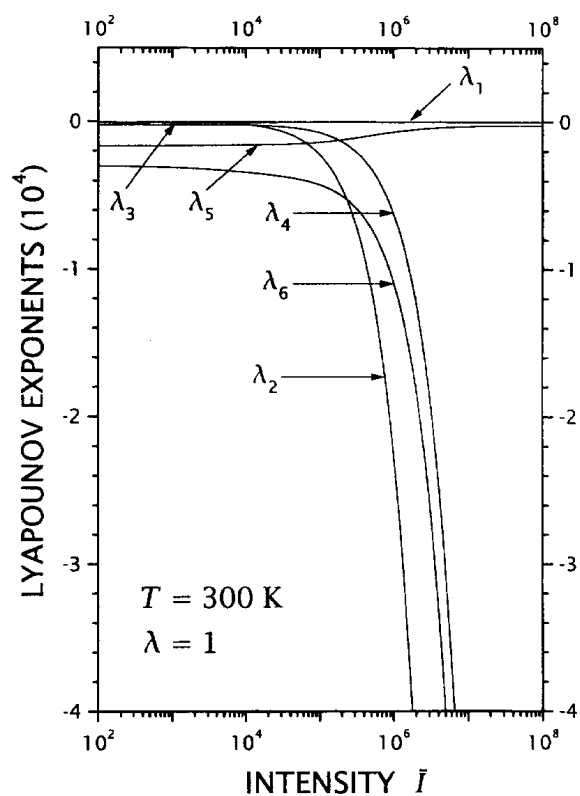

Figure 25. Lyapunov exponents in the steady state for the set of modes of Fig. 3 .

Moreover, Fig. 26 shows the sum of the Lyapunov exponents, namely the divergence of the supervector corresponding to the different contributions of the evolution operator [Cf. Eq. (69)], which is negative and increasing in modulus with the pumping intensity. This points to the fact that the trajectories are winding towards an attractor consisting in the Fröhlich-Bose-Einstein-like condensation. Hence, this indicates an increasing contraction of the elementary volumes in the space of thermodynamic states with increasing intensity of the pumping source. This is related to the interesting fact that the condensation becomes more and more effective, in the sense that the ratio, for each intensity, between the number of excitations in the condensate and the total number is increasing with $\bar{I}$, what is shown in Fig. 27.
Again there is a similarity with a Bose-Einstein condensation of bosons in equilibrium where the number of particles in the condensate increases in that case with decreasing temperature.

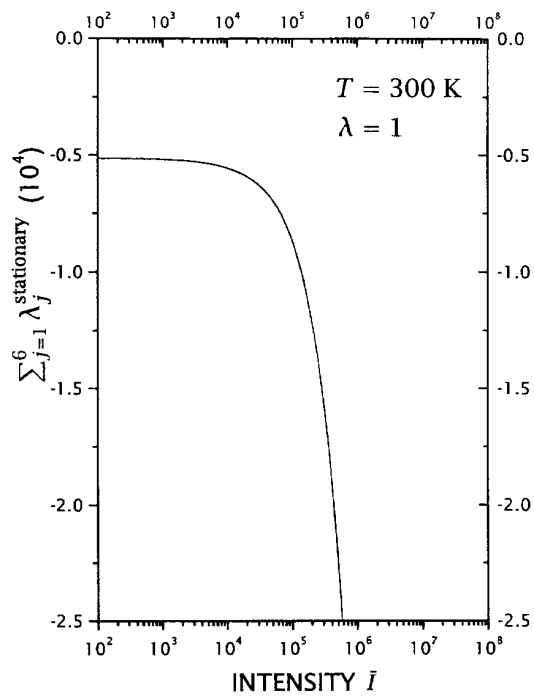

Figure 26. Sum of Lyapunov exponents or divergence of the evolution operator in the steady state for the set of modes of Fig. 2.

We notice that in Fröhlich condensate, with all Lyapunov exponents being negative, Kolmogorov entropy [96] is null, and then, as noted before, this implies that the trajectories are stable, the system does not produce information by itself (no selforganization follows) as would be for positiveness of some Lyapunov coefficient, but the system shows loss of information as evidenced by the increase in time of the informational entropy. However, as described by the order parameter of Fig. 21, with increasing nonlinearity (increasing values of $\lambda$ ) the informational entropy, for a giving pumping intensity, diminishes implying in smaller loss of information as ordering increases in the form of a more "dense" Fröhlich condensate, as already noticed.

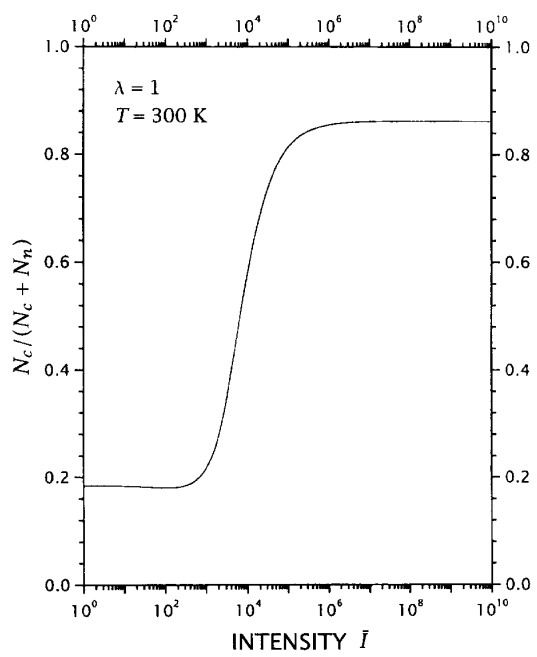

Figure 27. Fraction of excitations in the steady state. 


\section{On Biowater and Bioelectret Roles in Microtubules}

It has been said that water in nature has drawn artists for millennia, and both its flow and its serenity have borne deep meaning for philosophers. Thales - the sixth century BC Helenistic philosopher - concluded that water is the ultimate substance, the principle, or element, of all things. Later on, the also Helenistic philosopher, Empedocles proposed that the complexities of creation required four elements instead of one, and the liquid state took its place among them as the element water. Among earthly liquids, water is the most prevalent, covering more than 70 percent of the planetary surface, and it is by far the most important for the scientific consensus is that life arises because water is a prime natural medium for chemical reactions. Its mobile molecules act to diminish the electromagnetic forces that link atoms together, freeing the atoms to combine chemically with other free-floating atoms. According to present thinking, only a watery environment such the sea could have supported the chain of chemical reactions that formed such elaborated compounds as chlorophyll, DNA and hemoglobin, and the presence of water is essential for all the ongoing chemical processes of life [98].

There is no completely satisfactory theory of water, say, a master equation allowing to describe all its properties. Recently, as an alternative stategy, many investigators have turned to computer models to sum up the actions of all the molecules in a sample of water to arrive at their total effects. As already noticed above, water, with its characteristic angular structure, is an extraordinary substance for a variety of reasons, and, for example, its excellent properties as a solvent allowed for the possibility of life on Earth. To understand how water affects the structures of biological molecules, we must first appreciate the distinctive properties of water itself. These properties stem from the unique structure of water and the way its structure allows water to "manage" the electric charges of other molecules [99].

It can be recalled that, a single water molecule has an esssentially tetrahedral geometry, with an oxygen atom at the center of the tetrahedron, hydrogen atoms at two of the four corners and clouds of negative charge at the other two corners. The clouds of negative charge result from the way in which the atomic structures of oxygen and hydrogen combines. The oxygen-hydrogen bond is roughly of $10^{-8} \mathrm{~cm}$, and the angle close to 105 degrees. Moreover, it can be characterized three "types" of water in biological systems, namely: (1) ordered water that surrounds and strongly interacts with biological macromolecules; (2) bulk water beyond; (3) buried water that can be found within the biological macromolecules.

A single cell contains billions of water molecules, with all the space not occupied by the biological macromolecules being filled with water. Human cells are, in fact, mostly water, with the human body composed by roughly 60 percent of water by weight. The importance of water in living processes derives not only from its ability to form hydrogen bonds with other water molecules but also from its capacity to interact with various types of biological macromolecules.
Because of its polar nature, water readily interacts with other polar and charged molecules, such as acids, salts, sugars, and the various regions of proteins and DNA.

On this, on the one side, the alpha helices found in most proteins (as in Fig. 1) can unfold in water because water molecules can replace the hydrogen bonds that normally hold the helix together. Water molecules make a bridge between an oxigen atom and a nitrogen-hydrogen group, which would normally bond in the folded helix. On the other hand, as has been noticed [99], water is integral to the structure of DNA. In the absence of water it appears that DNA is unstable because of repulsive forces between the negatively charged phosphate groups that make up the backbone of the DNA double helix causing the molecule to rapidly break up. The presence of water apparently stabilizes the double-helical struture by forming hydrogen bonds with the phosphate groups. Seemingly, water molecules are able to interact with nearly every part of DNA's double helix, including the base pairs that constitute the genetic code.

Thus, it appears that water molecules can penetrate deeply into DNA's double helical structure and stabilize it. However, in contrast, water is not able to penetrate deeply into the structures of proteins, whose hydrophobic regions are tucked on the inside into a close-fitting core. Accordingly, the relation water-protein is centered on the protein surface.

Now, what can be the role of "biowater" in microtubules? As noted in the Introduction there exists a layer (of roughly $3 \mathrm{~nm}$ ) of ordered water in contact with the tubulin molecules in the interior of the microtubules, and along the columns of tubulin dimers [26].

At present, in view of the previous discussion, we can only conjecture that, on the one side the layer of ordered water can give stability to the columns of tubulin dimers. On the other side it may provide an appropriate environment for acting on as "catalyst" for the emergence of Fröhlich's effect. We recall that, as shown in the previous sections, it is fundamental for the quantum-coherent phenomenon of Fröhlich-Bose-Einstein condensation to follow the presence of a surrounding media, involved in the appearance of nonlinear quantum-kinetic equations as a sine qua non condition for such complex behavior to arise. Hence, if that is so, biowater would be basic for providing a medium in which quantum-coherent phenomena eventually associated to conscious processes in the brain — in Penrose's sense - can emerge.

Another relevant point is the possibility of formation of a bioelectret state in Fröhlich's condensate.

Proteins are polymers of amino acids: the 20 natural amino acids divide themselves in hydrophilic and hydrophobic classes. Thus, in general, naturally ocurring proteins contain dipolar residues, and can in principle be induced into the electret state. The electret effect has been found in many proteins, fibrous and globular, for example collagen and gelatin. Enzymes are also proteins, and important ones such as trypsin and urease exhibit the electret state.

Besides proteins there are other classes of important biopolymers for which the electret state has been found; it can be mentioned polysaccharides and polynucleotides. In 
the case of DNA there is indications of the existence of polarization storage sources.

In the case of enzymes water hydrates the protein in a solid icelike phase, and this phase has different energy levels depending on the orientation of the water dipole, leading to an electret-like behavior. On this it may be noticed the question of whether there is a form of normal ice in which the molecular dipoles have a net orientation, that is, the debate about whether such "ferroelectric" ice exists [100].

The question is now if systems displaying Fröhlich's effect can exhibit an electret state. If so it needs be analyzed the possible occurrence of ordered spatial states of dipoles resembling either ferroelectric, antiferroelectric, or helielectric states.

As we have seen in the previous sections the uniform in space Fröhlich's condensate appears as a stable state. Instability towards the formation of the ordered charge-density state would be indicated if the quasi-chemical potential of the mode lowest in frequency acquires the value of this frequency (and then the population of the mode diverges as in the usual Bose-Einstein condensation). This indicates that if the lowest frequency is at the zone center would follow a ferroelectric-like order, and if it is at the zone boundary would follow an antiferroelectric-like ordering.

More complex electret states can arise, once the case has a certain similarity to the existence of steady state chargedensity waves in an electron fluid, as discussed in [101], with a first bifurcation leading to the formation of the charge density wave followed by an ever increasing space organization leading to chaotic-like behavior (as in the case of chemical reactions [102]).

To analyze this question it is necessary to go outside of a thermodynamic description which is uniform in space, to a one that incorporates, besides the quantities of Eq. (1), nondiagonal quantities as $\hat{n}_{q \vec{Q}}=a_{\vec{q}+\frac{1}{2} \vec{Q}}^{\dagger} a_{\vec{q}-\frac{1}{2} \vec{Q}}$, whose average values over the nonequilibrium ensemble formalism produce the basic variables for describing a nonlinear quantum generalized thermo-hydrodynamics. One needs to analyze the normal modes and look for the possible "freezing" of one or more of these modes corresponding to the formation of steady state charge-density waves, much in the way as done in [101]. It may be the case that higher order nonlinear terms would need be included, which can give rise to fourth-order terms in the polarization field. This study is in the making [103].

\section{Concluding Remarks}

As it has been stressed in Section I, nowadays it is gaining ground the idea that Biology, Physics, Chemistry, Information Theory, and Complexity Theory need to joint forces to deal with questions as the origin of life and its evolution, the problem of a science of consciousness, and others in the life sciences.

Paul Davies [104] has maintained that solving the mystery of biogenesis is not just another problem on a long list of just-do scientific projects. Like the origin of the universe and the origin of consciousness, it represents something al- together deeper, because it tests the very foundations of our science and our world-view.

Also, "even though life is a physico-chemical phenomenon, its distinctiveness lies not in the strict physics and chemistry. The secret of life comes instead from its informational properties; a living organism is a complex information-processing system [emphasis is ours]. Hence, the ultimate problem of biogenesis is where biological information came from. Whatever remarkable the chemistry that may have occurred on the primeval earth or some other planet, life was sparked not by a molecular maelstrom as such but - somehow! - by the organization of information". [104].

In what refers to the processes governing consciousness in the human brain, Roger Penrose seems to have argued along a similar direction, as have been noticed in previous sections, in a kind of, say, a large-scale quantum action in brain functioning [26]. According to him one may expect a kind of quantum coherence - we would say an organization of information - in the sense that we must not look simply to the quantum effects of single particles, atoms, or even small molecules, but to the effects of quantum systems that retain their manifest nature at a much larger scale. We must look for something different as the appropriate type of controlling "mechanism" that might have relevance to actual conscious activity. Also, such processes must be the result of some reasonably large-scale quantum-coherent phenomenon, but coupled in such subtle way to macroscopic behavior, so that the system is able to take advantage of whatever is this particular physical process - as we have argued in the past sections involving a particular organization of information in Davies' sense.

At this point we reproduce Penrose's statement that, "Such a feat would be a remarkable one - almost an incredible one - for Nature to achieve by biological means. Yet I believe that the indications must be that she has done so, the main evidence coming from the fact of our own mentality. There is much to be understood about biological systems and how they achieve their magic".

This question of large-scale quantum coherence and connection with macroscopic order has been, in some sense, partially anticipated by Herbert Fröhlich in his "The Connection between Macro- and Microphysics" [12], in relation with supercondutivity and superfluidity. He pointed to the need to bridge the gap between the two levels introducing appropriate concepts, bringing together the completely systematic microscopic theory with the apparently somehow unsystematic macroscopic theory. Later on - or almost contemporaneously — Fröhlich further applied the ideas to the case of functioning of membranes, and giving rise to the idea of what we have called in previous sections of Fröhlich's effect (also - not so correctly - referred to as a nonequilibrium Bose-Einstein-like condensation).

This phenomenon has been called upon by Roger Penrose and other people as possibly having a role in consciousness, in connection with its eventual presence in microtubules in neurons. The role of these microtubules in the cytoskeleton of neurons in that connection may be reinforced by the fact that in patients with Alzheimer decease in whom 
ceases the flow of ideas, emotions, and memories, the disease - at least in one of the theories held - may be the result of the disruption of the microtubules as a consequence of misshaps in the so-called tau molecules which hold the microtubules in shape (Fig. 28).

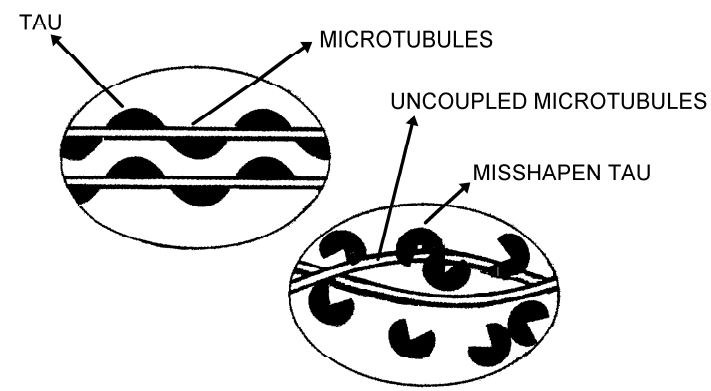

Figure 28. Disruption of the microtubules as a consequence of misshaps in the so-called tau molecules which hold the microtubules in shape.

Assuming that the phenomenon could have such a role, we have here reviewed in general terms, but in a certain depth, Fröhlich's effect. Such review has covered aspects of the phenomenon studied and discussed in terms of a, say, macro/micro-description, that is, in terms of a statistical thermo-mechanics for nonequilibrium systems. It is clearly evidenced the organization of information called for by Davies, and quite in accord with his statement that complexity and information can be illuminated by the subject of thermodynamics, a branch of science. In this case there is an answer for Davies query, already stated before, of where biological information comes from.

Also it makes contact with Davies' statements that, of couse, "organisms must comply with the laws of physics and chemistry, but these laws are only incidental to biology. Their main role is to permit an appropiate logical and informational system to come into being. Where chemical reactions are easy and thermodynamically favored, life will cheerfully make use of them, but if life needs to perform "unnatural" chemistry, it finds a way. The key step that was taken on the road to biogenesis was the transition from a state in which molecules slavishly follow mundane chemical pathways, to the one in which they organize themselves to forge their own pathways. Life opt out of the structures of chemistry by employing an informationcontrol channel, freeing it to soar above the clodlike thunderings of atomic interactions and create a new, emergent world of autonomous agency" [104].

As we have seen in the case of Fröhlich's effect, there occurs the emergence of complex behavior of bosons, as a result of exploring nonlinearities in the kinetic equations of evolution. There is a kind of autocatalytic process leading to synergetic ordering, and as a consequence a decreasing in informational entropy (maybe, uncertainty of information), following the fact of the consolidation of a long-range coherent macrostate. We see here the working of the microphysics - through the equations of quantum mechanics - with a subtle coupling to macrophysics through the resulting nonlinear kinetic equations, which are the average of the former over the nonequilibrium ensemble describing the expected behavior of the whole assembly of degrees of freedom of the system.

As closing remarks, we can now recall the sentence of the great Ludwig Boltzmann: "Thus, the general struggle for life is neither a fight for basic material ... nor for energy ... but for entropy [we say now information] becoming available by the transition from the hot sun to the cold earth" [105].

\section{References}

[1] E. Schrödinger, What Is Life? : The Physical Aspect of the Living Cell, Cambridge University, London, 1944.

[2] S. Mascarenhas, What is Biophysics?, in McMillan Encyclopaedia of Physics, edited by editor?, page pages?, Publisher?, City?, 19??

[3] J. Glanz, Physicists advance into Biology, Science 272, 646 (1996).

[4] W. Heisenberg, The end of Physics?, in Across the Frontiers, edited by R. N. Anshen, pages 184-91, Harper and Row, New York, 1975, Translation of article in Süddeutsche Zeitung, October 6, 1970.

[5] I. Prigogine and I. Stengers, Order out of the Chaos: Man's New Dialogue with Nature, Bantam, New York, 1984.

[6] G. Nicolis and I. Prigogine, Self-organization in Nonequilibrium Systems, Wiley-Interscience, New York, 1977.

[7] P. Davies, The New Physics: a synthesis, in The New Physics, edited by P. Davies, Cambridge Univ. Press, Cambridge, 1989.

[8] P. W. Anderson, More is different, Science 177, 393 (1972).

[9] L. von Bertalanffy, General Systems Theory, Braziller, New York, third edition, 1968.

[10] P. Glansdorff and I. Prigogine, Thermodynamic Theory of Structure, Stability, and Fluctuations, Wiley-Interscience, New York, 1971.

[11] I. Prigogine, Structure, Dissipation, and Life, in From Theoretical Physics to Biology, edited by M. Marois, North Holland, Amsterdam, 1969.

[12] H. Fröhlich, The connection between macro- and microphysics, Rivista del Nuovo Cimento 3, 490 (1973).

[13] H. Haken, Synergetics, Springer, Berlin, 1978.

[14] S. Kauffman, The Origins of Order: Self-organization and selection in evolution, Oxford Univ. Press, New York, 1993.

[15] L. Chong and L. B. Ray, Whole-istic Biology, and Introduction to Systems Biology Section, Science 295, 1661 (2002).

[16] H. Kitano, Systems Biology: A Brief Overview, Science 295, 1662 (2002).

[17] S. Kauffman, At Home in the Universe, Oxford Univ. Press, New York, 1995.

[18] P. W. Anderson, The Reverend Thomas Bayes, Needles in Haystacks, and the fifth force, Phys. Today 45(1), 9 (1992).

[19] E. T. Jaynes, Predictive Statistical Mechanics, in Frontiers of Nonequilibrium Statistical Physics, edited by G. T. Moore and M. O. Scully, pages 33, Plenum, New York, 1986.

[20] H. Jeffrey, Scientific Inference, Cambridge Univ. Press, Cambridge, third edition, 1973. 
[21] H. Jeffrey, Probability Theory, Clarendon, Oxford, 1961.

[22] A. Hobson, Irreversibility and Information in Mechanical Systems, J. Chem. Phys. 45, 1352 (1966).

[23] E. T. Jaynes, Information Theory and Statistical Mechanics I, Phys. Rev. 106, 620 (1957).

[24] E. T. Jaynes, Information Theory and Statistical Mechanics II, Phys. Rev. 108, 171 (1957).

[25] R. Luzzi, A. R. Vasconcellos, and J. G. Ramos, Statistical Foundations of Irreversible Thermodynamics, Teubner Bertelsmann-Springer, Berlin, 2000.

[26] R. Penrose, Shadows of the Mind: A Search for the Missing Science of Consciousness, Oxford Univ. Press, Oxford, 1994.

[27] H. Fröhlich, Quantum Mechanical Concepts in Biology, in From Theoretical Physics to Biology, edited by M. Marois, pages 13-22, North Holland, Amsterdam, 1969.

[28] D. Fröhlich, Notes and articles in honor of Herbert Fröhlich, in Cooperative Phenomena, edited by H. Haken and M. Wagner, Springer, Berlin, 1973.

[29] M. V. Mesquita, A. R. Vasconcellos, and R. Luzzi, Selective Amplification of Coherent Polar Vibrations in Biopolymers, Phys. Rev. E 48(5), 4049 (1993).

[30] S. Mascarenhas, Bioelectrets: Electrets in Biomaterials and Biopolymers, in Electrets, edited by G. M. Sessler, pages 321-46, Springer, Berlin, 1987.

[31] N. Grant and G. J. Hyland, Preprints of the main talks at the Microwave Application Workshop, to be held in the University of São Paulo (São Paulo, Brazil, tentatively in the Autumn (Southern Hemisphere) of 1998), (1998), We thank Dr. Norman Grant (Matra-Marconi Space-United Kingdom) and Prof. Oswaldo Massambani (São Paulo Univ.) for calling our attention to the Workshop, and providing us in advance the preprints of the invited talks.

[32] P. F. Miller and H. A. Gebbie, Laboratory millimeter wave measurements of atmospheric aerosols, Int. J. Infrared Milli. 17(10), 1573 (1996).

[33] A. S. Davydov, Biology and Quantum Mechanics, Pergamon, Oxford, 1982.

[34] A. R. Vasconcellos, M. V. Mesquita, and R. Luzzi, Statistical thermodynamic approach to vibrational solitary waves in acetanilide, Phys. Rev. Lett. 80(9), 2008 (1998).

[35] J. Lu and J. F. Greenleaf, Nondiffracting X-waves, IEEE Trans. Ultrason. Ferroelect. Freq. Contr. 39, 19 (1992).

[36] D. Snoke, Coherent exciton waves, Science 273, 1351 (1996).

[37] A. Mysyrowicz, E. Benson, and E. Fortin, Directed Beam of Excitons Produced by Stimulated Scattering, Phys. Rev. Lett. 77(5), 2844 (1996).

[38] A. R. Vasconcellos, M. V. Mesquita, and R. Luzzi, "Excitoner": Stimulated amplification and propagation of excitons' beams, Europhys. Lett. 49(5), 637 (2000).

[39] S. R. Hameroff and R. Penrose, Orchestrated reduction of quantum coherence in brain microtubules - a model of consciousness, in Towards a Science of Consciousness, edited by S. R. Hameroff, A. Kaszniak, and A. Scott, MIT Press, Cambridge, MA, 1996.
[40] S. R. Hameroff and R. C. Watt, Information-processing in microtubules, J. Theor. Biol. 98(4), 549 (1973).

[41] H. Biltz, H. Bütner, and H. Fröhlich, Electret model for the collective behavior of biological systems, Z. Naturforsch. B 36, 206 (1981).

[42] M. Eguchi, Title?, Phil. Mag. 49, 178 (1925).

[43] O. Heaviside, Electrical Papers, McMillan, London, 1892.

[44] B. Gross, Charge Storage in Solid Dielectrics, Elsevier, Amsterdam, 1964.

[45] A. C. Scott, F. Y. F. Chu, and D. W. McLaughlin, The Soliton: A new concept in applied Science, Proc. IEEE 61, 1443 (1973).

[46] J. Scott-Russell, Report on Waves, Proc. Roy. Soc. Edinburgh , 319 (1844).

[47] T. Beardsley, Plastic Power, Sci. Am. 276(4), 39 (1997).

[48] A. J. Heger, S. Kivelson, J. R. Schrieffer, and W. P. Su, Solitons in Conducting Polymers, Rev. Mod. Phys. 60, 781 (1988).

[49] T. Anderson and S. Roth, Conducting Polymers: Electrical Transport and Current Applications, Braz. J. Phys. 24(3), 746 (Septemper 1994).

[50] H. A. Haus, Molding light into solitons, IEEE Spectrum (March Issue), 48-53 (March 1993).

[51] A. S. Davydov, Solitons in Biology, in Solitons, edited by S. E. Trullinger, V. E. Zakharov, and V. L. Prokovsky, chapter 1, pages 1-51, Elsevier, New York, 1986.

[52] A. C. Scott, Davydov's Soliton, Phys. Rep 217, 1 (1992).

[53] M. V. Mesquita, A. R. Vasconcellos, and R. Luzzi, Neardissipationless Coherent Excitations in Biosystems, Int. J. Quantum Chem. 60, 689 (1996).

[54] M. V. Mesquita, A. R. Vasconcellos, and R. Luzzi, Solitons in highly excited matter: Dissipative thermodynamics and supersonic effects, Phys. Rev. E 58(6), 7913 (1998).

[55] D. N. Zubarev, Nonequilibrium Statistical Thermodynamics, Consultants Bureau, New York, 1974, [Neravnovesnaia Statisticheskaia Termodinamika (Izd. Nauka, Moscow, 1971)].

[56] D. N. Zubarev, V. N. Morozov, and G. Röpke, Statistical Mechanics of Nonequilibrium Processes. Vol. 1: Basic Concepts, Kinetic Theory; Vol. 2: Relaxation and Hydrodynamic Processes, Akademie Verlag-Wiley VCH, Berlin, 1996, 1997.

[57] R. Luzzi and A. R. Vasconcellos, On the Nonequilibrium Statistical Operator Method, Fortschr. Phys./Prog. Phys. 38, 887 (1990).

[58] R. Luzzi, A. R. Vasconcellos, and J. G. Ramos, Predictive Statistical Mechanics: A Nonequilibrium Ensemble Formalism, Kluwer Academic, Dordrecht, 2002.

[59] J. G. Ramos, A. R. Vasconcellos, and R. Luzzi, A Classical Approach in Predictive Statistical Mechanics: A Generalized Boltzmann Formalism, Fortschr. Phys./Prog. Phys. 43, 265 (1995).

[60] L. Lauck, A. R. Vasconcellos, and R. Luzzi, A Nonlinear Quantum Transport Theory, Physica A 168, 789 (1990). 
[61] J. R. Madureira, A. R. Vasconcellos, R. Luzzi, J. CasasVazquez, and D. Jou, Evolution of dissipative processes in a statistical thermodynamic approach II. Thermodynamic properties of a fluid of bosons, J. Chem. Phys. 108, 7580 (1998).

[62] J. R. Madureira, L. Lauck, A. R. Vasconcellos, and R. Luzzi, Markovian kinetic equations in a nonequilibrium statistical ensemble formalism, Phys. Rev. E 57, 3637 (1998).

[63] V. P. Kalashnikov, Equations of motion, green-functions, and thermodynamic relations in theories of linear relaxation with various sets of macroscopic variables, Theor. Mat. Phys. 35(1), 362 (1978).

[64] K. Walyazek, D. N. Zubarev, and A. Z. Kuzemskii, Schrödinger-type Equation with Damping for a Dynamical System in a Thermal Bath, Theor. Math. Phys. 5, 2 (1971).

[65] A. F. Fonseca, M. V. Mesquita, A. R. Vasconcellos, and R. Luzzi, Informational-statistical thermodynamics of a complex system, J. Chem. Phys. 112(9), 3967 (2000).

[66] P. T. Landsberg, Photons at non-zero chemical potential, J. Phys. C: Solid State Phys. 14, L1025 (1981).

[67] D. Kim and P. Y. Yu, Phonon Temperature Overshot in GaAs excited by picosecond laser pulses, Phys. Rev. Lett. 64, 946 (1990).

[68] A. C. Algarte, A. R. Vasconcellos, and R. Luzzi, Ultrafast phenomena in the photoinjected plasma in semiconductors, Braz. J. Phys. 26, 543 (1996).

[69] L. D. Landau and E. M. Lifshitz, Electrodynamics of Continuous Media, Pergamon, Oxford, 1960.

[70] H. Haken, Handbuch der Physik XXV/2C, Springer, Berlin, 1970.

[71] V. E. Zakharov, The Inverse Scattering Method, in Solitons, edited by R. K. Bullough and P. J. Caudrey, chapter 7, pages 243-85, Springer, Berlin, 1980.

[72] G. Careri, U. Buontempo, F. Carta, E. Gratton, and A. C. Scott, Infrared Absorption in Acetanilide by Solitons, Phys. Rev. Lett. 51(4), 304 (1983).

[73] J. L. Sauvajol, R. Almaraic, J. Moret, M. Barthes, and J. L. Ribet, Temperature Dependence of the Raman spectrum of fully deuterated acetanilide, J. Raman Spectrosc. 20, 517 (1989).

[74] G. Careri and E. Gratton, Fine Structure of the Amide I Band in Acetanilide, Phys. Rev. A 37(10), 4048 (1988).

[75] M. Sakai, N. Kuroda, and Y. Nishima, High-pressure Raman Study of Vibrational Spectra in Crystalline Acetanilide, Phys. Rev. B 47(1), 150 (January 1993).

[76] M. Barthes, R. Almaraic, J. L. Sauvajol, , R. Currat, J. Moret, , and J. L. Ribet, Neutron scattering investigation of deuterated crystalline acetanilide, Europhys. Lett. 7, 55 (1988)

[77] M. Barthes, J. Eckert, S. W. Johnson, J. Moret, B. I. Swanson, and C. J. Unjeker, Anomalous Vibrational Modes in acetanilide as studied by inelastic neutron scattering, J. Phys. I France 2, 1929 (1992).

[78] R. Luzzi and A. R. Vasconcellos, Response function-theory for far-from-equilibrium statistical systems, J. Stat. Phys. 23(5), 539 (1980).
[79] V. P. Kalashnikov, Response to a Mechanical Perturbation and the Green's Functions for Nonequilibrium Systems, Theor. Math. Phys. 9, 1003 (1971).

[80] M. V. Mesquita, A. R. Vasconcellos, and R. Luzzi, Optical and Acoustical Solitary Waves: Thermodynamic and "Superluminal” Effects, Phys. Rev. Lett. (1997), submitted.

[81] C. Rasinariu, Solitons of KdV Equation, URL: —http://www.mapleapps.com/categories/science/ physics/html/solKdV.html一.

[82] V. L. Ginsburg, Certain Theoretical Aspects of Radiation due to Superluminal Motion in a Medium, Soviet Phys.Uspekhi 2, 874 (1960).

[83] V. M. Agranovich and V. L. Ginsburg, Spatial Dispersion in Crystal Optics and the Theory of Excitons, WileyInterscience, London, 1966.

[84] P. Kocevar, Hot phonon dynamics, Physica B 34, 155 (1985).

[85] H. Fröhlich, The Biological Effects of Microwaves and Related Questions, in Advances in Electronics and Electron Physics, volume 17, pages 85-152, Academic Press, New York, 1980.

[86] A. C. Algarte, A. R. Vasconcellos, and R. Luzzi, Kinetic of hot Elementary Excitations in Photoexcited Polar Semiconductors, Phys. Stat. Sol. (b) 173, 487 (1992).

[87] J. Lu, Z. Hehong, and J. F. Greenleaf, Biomedical ultrasound beam forming, Ultrasound Med. Biol. 20, 403 (1994).

[88] W. A. Rodrigues and J. Lu, On the Existence of Undistorted Progressive Waves, Found. Phys. 27, 435 (1997), We thank W. A. Rodrigues and J. A. Majorino for providing us with a postscript file of this figure.

[89] R. S. Knox, in Theory of Excitons, edited by F. Seitz and J. Turnbull, number Solid State Physics Series, Suppl. 5, Academic Press, New York, 1963.

[90] H. Fröhlich, Long Range Coherence and the Action of Enzymes, Nature 228, 1093 (1970).

[91] A. R. Vasconcellos, A. C. Algarte, and R. Luzzi, Diffusion of Photoinjected Carriers in Plasma in Nonequilibrium Semiconductors, Phys. Rev. B 48(15), 10873 (1993).

[92] J. M. Ziman, Electrons and Phonons, Clarendon, Oxford, 1960.

[93] E. Hanemura and H. Hang, Qual?, Phys. Rep. 33, 209 (1977).

[94] D. Marcuse, Theory of Dielectric Optical Waveguides, Academic, Boston, 1991.

[95] M. A. Tenan, A. R. Vasconcellos, and R. Luzzi, Mechanostatistical Foundations for Generalized Nonequilibrium Thermodynamics, Fortschr. Phys./Prog. Phys. 47, 1 (1996).

[96] G. Nicolis, Introductory remarks: Thermodynamics today, Physica A 213, 1 (1995).

[97] G. Nicolis and D. Daems, Probabilistic and thermodynamic aspects of dynamical systems, Chaos 8(2), 311 (1998).

[98] S. Perkowitz, The Rarest Element, Sciences 39(1), 34 (JanFeb 1999).

[99] M. Gerstein and M. Levitt, Simulating Water and the Molecules of Life, Sci. Am. 275(5), 100 (November 1998). 
[100] S. T. Bramwell, Condensed-matter science: Ferroelectric ice, Nature 397, 212 (1999)

[101] S. A. Hassan, A. R. Vasconcellos, and R. Luzzi, NonlinearDriven Instability of Dynamical Plasma in Solids: Emergence of Spacially Self-Organized Order and Chaotic-Like Behavior, Eur. J. Phys. B 13(1), 131 (2000).

[102] G. Nicolis and I. Prigogine, Exploring Complexity, Freeman, New York, 1989.
[103] M. V. Mesquita, A. R. Vasconcellos, S. Mascarenhas, and R. Luzzi, Title?, unpublished.

[104] P. C. Davies, The Fifth Miracle: The Search for the Origin and Meaning of Life, Simon \& Schuster, New York, 1999.

[105] L. Boltzmann, Populare Schriften, Leipzig, 1905, cited in K. Mainzer, Thinking in Complexity, Springer, Berlin, 1994. 